\title{
Size Dependence of Yield Strength Simulated by a Dislocation-density Function Dynamics Approach
}

\author{
P.S.S. Leung, H.S. Leung, B. Cheng and A.H.W. Ngan ${ }^{\S}$ \\ Department of Mechanical Engineering, The University of Hong Kong, \\ Pokfulam Road, Hong Kong \\ ${ }^{\S}$ Correspondence Author (email: hwngan@hku.hk)
}

\begin{abstract}
The size dependence of the strength of nano- and micron-sized crystals is studied by a new simulation approach in which the dynamics of the density functions of dislocations are modeled. Since any quantity of dislocations can be represented by a density, this approach can handle large systems containing high quantities of dislocations, which may handicap discrete dislocation dynamics schemes due to the excessive computation time involved. For this reason, pillar sizes spanning a large range, from the submicron to micron regime, can be simulated. The simulation results reveal the power-law relationship between strength and specimen size up to a certain size, beyond which the strength varies much more slowly with size. For specimens smaller than $\sim 4000 \mathrm{~b}$, their strength is found to be controlled by the dislocation depletion condition, in which the total dislocation density remains almost constant throughout the loading process. In specimens larger than $\sim 4000 b$, the initial dislocation distribution is of critical importance since the presence of dislocation entanglements is found to obstruct deformation in the neighboring regions within a distance of $\sim 2000 \mathrm{~b}$. This length scale suggests that the effects of dense dislocation clusters are greater in intermediate sized specimens (e.g $4000 b$ and $8000 b$ ) than in larger specimens (e.g. 16000b), according to the weakest-link concept.
\end{abstract}

Keywords: crystal plasticity, dislocations, size effect, tensile test 


\section{Introduction}

The mechanical strength of nano- and micron-sized single crystals has attracted continuous interest for more than half a century. Brenner $(1956,1957)$ first observed that copper and silver whiskers exhibited extremely sharp and high yield points close to the theoretical strength. In recent years, contemporary fabrication techniques, including focused ion beam (FIB) and direct deposition methods, have been used to produce micro-specimens with more precisely adjustable sizes (Uchic et al. 2004; Greer et al. 2005, Kiener et al. 2008; Kim et al. 2009; Greer and De Hosson 2011), and these efforts are augmented by in situ transmission electron microscopy (TEM) investigations (De Hosson et al. 2006, Legros et al. 2008, Shan et al. 2008, Oh et al. 2009) to provide a more complete picture of the dislocation mechanisms responsible. Nano- or micron- sized crystals in general are found to deform in a jerky manner with stochastic occurrence of discrete strain bursts (Uchic et al. 2004, Ng and Ngan 2008b, Shan et al. 2008, Kim et al. 2009, Kim et al. 2012). In addition, their yield strength $\sigma$ in general exhibits a remarkable smaller-being-stronger size effect according to a power law $\sigma \sim D^{-m}$, where $D$ is specimen size (Greer and Nix 2006; Dou and Derby 2009). The exponent $m$ here was found to fall within a range of about 0.2 to 1 . For example, $m$ was reported to be 0.69 for $\mathrm{Cu}, 0.89$ for $\mathrm{Al}$, 0.47 for [111] Ta (Kaufmann et al. 2011), 0.22 for [001] Mo and 0.34 for [235] Mo (Schneider et al. 2009). For Mo, Kim et al. (2012) also reported $m$ to be 0.39 and 0.53 respectively in compression and tension along [001], and 0.57 and 0.60 respectively in compression and tension along [011]. However, for $\mathrm{Au}$, no tension-compression asymmetry was observed (Kim and Greer 2009). For Al, $m$ was found to be pre-strain dependent, dropping from 0.98 for pristine samples to 0.52 for $15 \%$ pre-strained samples (Gu and Ngan 2013).

One explanation proposed for the smaller-being-stronger size effect and jerky deformation is easy dislocation depletion from the small specimen and the need for re-nucleation (Greer and Nix 2006). This so-called dislocation starvation state has been confirmed in submicron crystals by in situ TEM experiments (Shan et al. 2008). For larger specimens in the micron regime where complete starvation is difficult to achieve, the size effect has been proposed to be a consequence of the shorter dislocation source length (Parthasarathy et al. 2007, Akarapu 2010), or finer dislocation mesh size (Gu and Ngan 2013), in smaller specimens. In particular, the exact power-law form $\sigma \sim D^{-m}$ has been shown to be a natural consequence of Taylor-type interactions in an existing dislocation network in which the mesh lengths obey a power-law, or fractal, distribution (Ngan 2011). In this case the exponent is given as $m=$ $3 /(q+n)$, where $q$ is the fractal dimension of the dislocation mesh and $n$ is the stress exponent of the dislocation velocity (Ngan 2011; Gu and Ngan 2013). Since $2 \leq q \leq 3$ for threedimensional fractal networks and $n$ is usually $\geq 1, m$ is bounded between 0 and 1 as is commonly observed.

Computer simulations based on discrete dislocation dynamics (DD) have also been employed extensively to understand small-scale plasticity (Devincre et al. 2006, Csikor et al. 
2007, Motz et al. 2009, Akarapu et al. 2010, Huang et al. 2012). 2D DD (Van der Giessen and Needleman 1995) considers only parallel dislocation lines, and hence ignores the interactions of intersecting dislocations as in Taylor hardening. On the other hand, 3D DD (Devincre and Condat 1992) considers 3D curved dislocations in a much more realistic way, but then the amount of computation involved would be greatly increased and for this reason, it is only applicable to systems containing low quantities of dislocation segments. Previous attempts using 3D DD were therefore limited to the small specimen size range of sub-micron to a few microns, with dislocation densities of $10^{12}$ to $10^{15} \mathrm{~m}^{-2}$ (Akarapu et al. 2010, Zhou et al. 2010, Huang et al. 2012). Moreover, the strain rates used in such 3D DD studies were usually of the order of $10^{2}$ $\mathrm{s}^{-1}$ or above (Motz et al. 2009, Akarapu et al. 2010, Zhou et al. 2010), which is many orders of magnitude higher than the typical strain rate of around $10^{-3} \mathrm{~s}^{-1}$ in real experiments. A compromised approach, the so-called 2.5D DD (Benzerga 2009, Gómez-García et al. 2006), has also been used which is a mesoscopic simulation scheme based on 2D DD but with certain 3D mechanisms, such as Taylor interactions and Frank-Read sources, partially considered in a highly simplified manner. Molecular dynamics have also been employed (Yamakov et al. 2002, Komanduri et al. 2001, Parrinello and Rahman 1981), but even with today's computing power, the micron-size regime is still a challenge and in all cases, the time scale that can be handled is still many orders of magnitude shorter than real situations.

To simulate dislocation plasticity over a wide range of specimen size from, say, a micron to tens or hundreds of microns in a unified way, a better technique is needed. We recently developed a new simulation methodology based on the dynamics of the density functions of dislocations, and applied it to study the strength of micron-size crystals (Leung et al. 2014). Since any quantity of dislocations can be represented by a density, a simulation scheme based on the dynamics of density can handle high amounts of dislocations, which may handicap 3D DD because of the excessive computation time needed. This new simulation scheme, called dislocation-density function dynamics (DDFD), has been shown to be able to capture dislocation pattern formation in mechanically vibrated crystals (Cheng et al. 2014). In our previous work (Leung et al. 2015), the power-law size dependence of strength is predicted and in this work, we focus on the detailed mechanisms leading to such a size effect.

\section{Simulation Methodology}

The simulation strategy is based on a coarse-graining or representative volume element (RVE) concept in which the dislocation microstructure is represented solely by density functions $\rho_{\alpha}(\vec{r}, t)$ that are continuously distributing over space $\vec{r}$ and time $t$. This implies that individual dislocation lines are not represented, although different dislocation types, represented by the index $\alpha$, are considered. The underlying assumption is that the entire dislocation microstructure can be meaningfully considered as being made up of discrete voxels, which are large enough so that the dislocations present inside each of them can be regarded as uniformly distributing in a 
featureless manner, and at the same time small enough compared to the length scales of the important features of the entire dislocation microstructure. Such a coarse-graining assumption has been the foundation of many previous dislocation-density based models of crystal plasticity (Busso et al. 2000, Dunne et al. 2007, Alankar et al. 2009, Chen et al. 2013), and here, we simply assume that it is valid for micron-sized deforming volumes.

Many dislocation-density based approaches for crystal plasticity have been proposed in the past (Ananthakrishna 2007), but our DDFD approach differs in terms of two fundamental aspects. First, most of the previous models focus on the evolution of geometrically necessary dislocations (GNDs) in response to the elastoplastic shape change of the crystal (Asaro and Rice 1977; Acharya 2001; Taupin et al. 2008; Puri, Das and Acharya 2011; Chen et al. 2013). In reality the evolution of the GNDs is coupled to that of the statistically stored dislocations (SSDs), but in these approaches, while the evolution of the GNDs is described by very rigorous elastoplastic frameworks, the SSDs are treated much less rigorously, as just to offer some empirical back stress (Groma, Csikor and Zaiser 2003; Arsenlis et al. 2004; Yefimov and Giessen 2005; Puri, Das and Acharya 2011; Hirschberger et al. 2011) or in other ad hoc manners (Busso et al. 2000, Rezvanian et al. 2007), or are simply ignored (Roy and Acharya 2005; Limkumnerd and Sethna 2006; Chen et al. 2013). On the contrary, as described below, our DDFD approach considers the full dynamics of all dislocations without the need to distinguish between them as GNDs or SSDs. All dislocations, both GNDs and SSDs, are subjected to the same velocity law for evolution from the effective glide stress acting on them, as is the case in discrete DD models as well as in reality. While no a priori assumption about GNDs or SSDs is needed, after the simulation, the effects played by the GNDs and SSDs can be extracted out from the results (Cheng et al. 2014). However, the mechanics framework adopted for the dislocation evolution is purely plastic, with elastic deformation either neglected as in this work, or incorporated additively to the plastic deformation as a first-order approximation (Leung et al. 2015).

Secondly, the mutual elastic interactions between dislocations are well-known to be important, and as shown by Hirth and Lothe (1992), two dislocation segments $d \vec{l}_{1}$ and $d \vec{l}_{2}$ of Burgers vectors $\vec{b}_{1}$ and $\vec{b}_{2}$ separated by a distance $R$ will experience a mutual interaction force $\delta \vec{f}$ given by

$$
\begin{aligned}
& \delta \vec{f}=-\frac{\mu}{8 \pi}\left[\left(\vec{b}_{2} \times \vec{b}_{1}\right) \cdot \vec{\nabla}\left(\nabla^{2} \mathrm{R}\right)\right]\left(d \vec{l}_{1} \times d \vec{l}_{2}\right)-\frac{\mu}{8 \pi}\left\{\left[\vec{b}_{2} \times \vec{\nabla}\left(\nabla^{2} \mathrm{R}\right)\right] \times d \vec{l}_{1}\right\}\left(\vec{b}_{1} \cdot d \vec{l}_{2}\right) \\
& -\frac{\mu}{4 \pi(1-v)}\left[\left(\vec{b}_{2} \times d \vec{l}_{2}\right) \cdot \vec{\nabla}\right]\left(d \vec{l}_{1} \times \vec{b}_{1} \mathbb{T}\right)+\frac{\mu}{4 \pi(1-v)}\left[\left(\vec{b}_{2} \times d \vec{l}_{2}\right) \cdot \vec{\nabla}\left(\nabla^{2} \mathrm{R}\right)\right]\left(d \vec{l}_{1} \times \vec{b}_{1}\right)
\end{aligned}
$$

where $\mu$ is the shear modulus and $v$ the Poisson ratio. While discrete DD approaches are fully compatible with eqn. (1), none of the dislocation-density based approaches proposed to-date contains description that is compatible to, or even approximate of, eqn. (1). As described below, the present DDFD approach takes into full consideration of the mutual elastic interactions 
according to eqn. (1), with Taylor interactions, generation, annihilation, cross-slip, and flux (conservative) motion, also taken into account.

Details of our DDFD scheme has been reported elsewhere (Leung et al. 2015) and in the following, only the key concepts are described.

\subsection{Dislocation densities and stresses for evolution}

In this paper, we consider a face-centered cubic model with 12 slip systems of the type $\frac{1}{2}<110>\{111\}$. In each slip system, the line-orientation space of the dislocations is discretized, and following Arsenlis et al. (2004), we discretize the orientation space into the minimum of four characters only, namely, positive edge $(\mathrm{e}+)$, negative edge (e-), positive screw $(\mathrm{s}+)$ and negative screw (s-), as illustrated in Figure 1. As a result, 48 dislocation density functions $\rho_{\alpha}(\vec{r}, t)$, with $\alpha=1,2, \ldots, 48$, are modelled. The various forms of glide stress that govern their evolution are described below.

(i) Long-range elastic interaction stress $\left(\tau_{\alpha}^{i n t}\right)$

As shown in Fig. 2, a unit dislocation segment along line direction $\hat{\xi}$ at $\vec{r}$ will experience a net Peach-Koehler glide stress $\tau_{\alpha}^{i n t}$ due to the density of dislocations $\rho_{\alpha^{\prime}}\left(\vec{r}^{\prime}, t\right)$ situated at each pixel point $\vec{r}^{\prime}$ in space. By generalizing eqn. (1) for discrete dislocation segments to densities of dislocations that are mutually interacting, $\tau_{\alpha}^{i n t}$ can be shown to be given by (Leung et al. 2015)

$$
\tau_{\alpha}^{i n t}\left(\vec{r}_{i}\right)=\sum_{\alpha^{\prime}=1}^{48} \sum_{j \neq i} g\left(\vec{r}_{j}-\vec{r}_{i}\right) \rho_{\alpha^{\prime}}\left(\vec{r}_{j}\right) V
$$

where $i$ indexes the voxel at which the Peach-Koehler stress is evaluated, $j$ indexes the neighbor voxels of $i$ within a prescribed interaction radius, $V$ is the voxel volume, and full expressions of the kernel $g\left(\vec{r}_{j}-\vec{r}_{i}\right)$ are given in Leung et al. (2015). The cutoff radius in this work was set to be $400 b$ which provides for a good compromise between accuracy and computational speed. It should be noted that, by virtue of the outer summation in eqn. (2), the density $\rho_{\alpha^{\prime}}\left(\vec{r}_{j}\right)$ at the source location $\vec{r}_{j}$ needed to be considered can simply be replaced by the net GND density, since the effects of all SSD should sum up to zero and hence can be ignored. However, the calculated $\tau_{\alpha}^{i n t}\left(\vec{r}_{i}\right)$ at the field point $\vec{r}_{i}$ applies to the corresponding dislocation type $\alpha$ there no matter whether the dislocations involved are GND or SSD. In practice, it will not be possible to tell which particular dislocation in given structure is a GND or SSD, and in the present approach, the dynamics of all dislocation contents in the structure, without the need to know which are GND and which are SSD, are considered. 
(ii) Taylor hardening resistance $\left(\tau_{\alpha}^{\text {Taylor }}\right)$

The forest dislocation densities inside a voxel impose a resistance stress to the glide of the dislocations in that voxel given by:

$$
\tau_{\alpha}^{\text {Taylor }}=\mu b \sqrt{\sum_{\alpha \prime}\left(\vec{n}_{\alpha} \cdot \vec{\xi}_{\alpha \prime}\right)^{2} \rho_{\alpha \prime}}
$$

where $b=|\vec{b}|, \mu$ is the shear modulus, $\vec{n}_{\alpha}$ is the slip plane normal for type $\alpha$, and $\vec{\xi}_{\alpha \prime}$ is the line direction of the forest dislocations. Eqn. (3) here is a reduced form of the general law for strain hardening, which would contain various interaction effects from co-planar as well as intersecting slip systems (Franciosi and Zaoui 1982). The actual interaction coefficients in the FranciosiZaoui scheme are, however, not known, and furthermore, much of the co-planar interactions has already been represented by the elastic interactions in eqn. (2). For these reasons, in the simplified form of the Taylor hardening in eqn. (3), only the effects of the intersecting slip systems are represented by the coefficients $\left(\vec{n}_{\alpha} \cdot \vec{\xi}_{\alpha \prime}\right)^{2}$, with coplanar interactions neglected.

(iii) Lattice Friction stress $\left(\tau_{\alpha}^{f}\right)$

In addition to the resistance stress from Taylor hardening considered above, the lattice friction or Peierls stress also opposes dislocation glide. In the present work, the lattice friction stress is taken simply as a constant value of $2 \times 10^{-3} \mu$.

(iv) Externally applied stress $\left(\tau_{\alpha}^{\text {ext }}\right)$

In this work, elastic deformation is neglected and so $\tau_{\alpha}^{e x t}$ arises directly from the resolved shear stress on the slip system for type $\alpha$. In order to implement constant strain rate conditions, however, the externally applied load is adjusted from time step to time step according to the difference of the actual strain rate from the prescribed value. One method is to adjust the stress in proportion to the error (El-Awady et al. 2009), i.e.

$$
\dot{\sigma} \propto\left(\dot{\varepsilon}_{\text {prescribed }}-\dot{\varepsilon}_{\text {actual }}\right)
$$

While this works pretty well in most cases, for smaller specimens where extensive dislocation depletion frequently occurs, serious overshoot in stress may occur whenever dislocation depletion and a tremendous drop in strain rate happen, so that the applied stress is increased 
rapidly. To alleviate such a problem, a derivative term similar to that used in proportionalintegral-differential control is added to the stress adjustment, i.e. the stress is now adjusted also in proportion to the rate of change of the strain-rate error. If the error in strain rate is decreasing, the stress adjustment is also decreased and vice versa. This is found to be effective in suppressing the stress overshooting.

In addition to the externally applied stress, image stresses which pull dislocations towards free surfaces are normally considered in discrete DD simulations (Fivel and Canova 1999). While it is not particularly difficult to include image stresses in the present work, this is not done because the voxel size in the present approach is typically hundreds of Burgers vectors large. Since image stresses attenuate from the free surface over distances that scale with $b$, the image stress acting even in the first voxel next to a free surface would be negligible. For this reason, image stresses are ignored in the present work.

The stresses discussed in (i) to (iv) above sum up to give the effective glide stress acting on type $\alpha$ dislocations as:

$$
\tau_{\alpha}^{\text {eff }}(\vec{r}, t)=\tau_{\alpha}^{\text {int }}(\vec{r}, t)-\tau_{\alpha}^{\text {Taylor }}-\tau_{\alpha}^{f}+\tau_{\alpha}^{\text {ext }}(\vec{r}, t)
$$

\subsection{Dislocation density evolution and strain rate}

As in discrete DD, the dislocation velocity of each type $\vec{v}_{\alpha}(\vec{r}, t)$ is assumed to depend on the effective stress $\tau_{\alpha}^{\text {eff }}$ above in a power law

$$
\overrightarrow{\mathrm{v}}_{\alpha}(\vec{r}, t)=\operatorname{sgn}\left(\tau_{\alpha}^{e f f}(\vec{r}, t)\right)(\hat{n} \times \hat{\xi}) v_{0}\left|\tau_{\alpha}^{e f f}(\vec{r}, t) / \tau_{0}\right|^{m}
$$

where $v_{0}, \tau_{0}$ and $m$ are constants, $\operatorname{sgn}(x)$ is the sign function, $\hat{\xi}$ is the unit vector along the dislocation line of type $\alpha$ dislocations and $\hat{n}$ is their slip plane normal.

The dislocation flux, which is the quantity (i.e. line length) of dislocations moving across a perpendicular surface of unit area per unit time, is given as:

$$
\overrightarrow{\mathrm{J}}_{\alpha}(\vec{r}, t)=\rho_{\alpha}(\vec{r}, t) \overrightarrow{\mathrm{v}}_{\alpha}(\vec{r}, t)
$$

The time-space evolution of type $\alpha$ dislocation density is then governed by the continuity law:

$$
\dot{\rho}_{\alpha}(\vec{r}, t)=-\vec{\nabla} \cdot\left[\rho_{\alpha}(\vec{r}, t) \overrightarrow{\mathrm{v}}_{\alpha}(\vec{r}, t)\right]+\text { net production rate }
$$


where the net production rate is the difference between dislocation generation and annihilation rates, which include the effects of generation by flux gradients (i.e. Frank-Read source type of generation), dipole annihilation, as well as cross-slip. These will be discussed later in section 2.3.

The instantaneous strain-rate field due to type $\alpha$ dislocations at time $t$ is $\rho_{\alpha}(\vec{r}, t)\left[\overrightarrow{\mathrm{v}}_{\alpha}(\vec{r}, t) \cdot(\hat{\mathrm{n}} \times \hat{\xi})\right](\vec{b} \otimes \hat{\mathrm{n}})=\left|\vec{J}_{\alpha}(\vec{r}, t)\right|(\vec{b} \otimes \hat{\mathrm{n}})$, where $\otimes$ is the tensor product operator of the two corresponding vectors. The gross strain-rate tensor field is obtained by summing up strain rates contributed by each individual type of dislocations:

$$
\dot{\varepsilon}(\vec{r}, t)=\sum_{\alpha}(\vec{b} \otimes \hat{\mathrm{n}})\left[\overrightarrow{\mathrm{J}}_{\alpha}(\vec{r}, t) \cdot(\hat{\mathrm{n}} \times \hat{\xi})\right]
$$

The cumulative strain tensor field at time $t$ is therefore

$$
\varepsilon(\vec{r}, t)=\int_{0}^{t} \dot{\varepsilon}\left(\vec{r}, t^{\prime}\right) d t^{\prime}
$$

To numerically solve eqn. (8), we made use of the finite volume method (FVM) which can deal with sharp gradients. In the FVM scheme, for a typical flux operator

$$
\dot{\rho}+\vec{\nabla} \cdot(\rho \vec{v})
$$

the first-order "upwind" discretization of the $i$-th voxel is

$$
\frac{d \rho_{i}}{d t}+\frac{1}{\Delta x_{i}}\left[(\rho v)_{i+1}-(\rho v)_{i}\right]+\frac{1}{\Delta y_{i}}\left[(\rho v)_{i+1}-(\rho v)_{i}\right]+\frac{1}{\Delta z_{i}}\left[(\rho v)_{i+1}-(\rho v)_{i}\right]
$$

To cater for dislocation densities with high gradients, the Monotone Upstream-centered Schemes for Conservation Laws (MUSCL), which are total variation diminishing (TVD) schemes (Wesseling 2001), are employed. In this work the Kurganov-Tadmor central scheme is also used, as is the case in earlier dislocation-based models (Chen et al. 2010, Choi et al. 2012, Chen et al. 2013). Using the voxel states of the previous time step, the MUSCL scheme computes the flux through the voxel walls on the left and right hand side. The flux-operator can then be re-written in the following semi-discrete form

where

$$
\frac{d \rho_{i}}{d t}+\frac{1}{\Delta x_{i}}\left[(\rho v)^{*}{ }_{i+1 / 2}-(\rho v)^{*}{ }_{i-1 / 2}\right]+\cdots
$$

$$
(\rho v)^{*}{ }_{i \pm 1 / 2}=\frac{1}{2}\left\{\left[\rho_{i \pm 1 / 2}^{R} v_{i \pm 1 / 2}+\rho_{i \pm 1 / 2}^{L} v_{i \pm 1 / 2}\right]-\max \left[\left|v_{i}\right|,\left|v_{i \pm 1}\right|\right]\left[\rho_{i \pm 1 / 2}^{R}-\rho_{i \pm 1 / 2}^{L}\right]\right\} ;
$$


$\rho_{i+1 / 2}^{L}=\rho_{i}+0.5 \phi\left(r_{i}\right)\left(\rho_{i+1}-\rho_{i}\right) ; \rho_{i+1 / 2}^{R}=\rho_{i+1}+0.5 \phi\left(r_{i+1}\right)\left(\rho_{i+2}-\rho_{i+1}\right) ;$

$\rho_{i-1 / 2}^{L}=\rho_{i-1}+0.5 \phi\left(r_{i-1}\right)\left(\rho_{i}-\rho_{i-1}\right) ; \quad \rho_{i-1 / 2}^{R}=\rho_{i}+0.5 \phi\left(r_{i}\right)\left(\rho_{i+1}-\rho_{i}\right) ;$

$r_{i}=\left(v_{i}-v_{i-1}\right) /\left(v_{i+1}-v_{i}\right)$.

Here, $\phi(r)$ is the flux limiter given as $\phi(r)=\left\{\begin{array}{l}0, \text { for } r<0 \text {; } \\ 1 \text {, for } r>1\end{array}\right.$

For the voxels exposing to a free surface, the voxel on either the left or right hand side would be missing. Therefore, "ghost cells" are used to mimic the neighboring voxels for these free-surface voxel, in order to effectively provide the necessary boundary conditions. In this work, the free surface adopts a no-entry, free-exit boundary condition by using ghost cells with velocity and density values assigned as follows:

For $i=1$ or $j=1$ or $k=1$ :

$$
\left\{\begin{array}{c}
v_{i-1}=\min (0, v i), \\
\rho_{i-1}=\rho_{i} \max \left(0, \operatorname{sgn}\left(-v_{i}\right)\right), \quad \text { and } \\
(\rho v)^{*}{ }_{i-1 / 2}=\min \left(0,(\rho v)^{*}{ }_{i-\frac{1}{2}}\right)
\end{array}\right.
$$

For $i=n x$ or $j=n y$ or $k=n z$ :

$$
\left\{\begin{array}{c}
v_{i+1}=\max (0, v i), \\
\rho_{i+1}=\rho_{i} \max \left(0, \operatorname{sgn}\left(v_{i}\right)\right), \\
(\rho v)^{*}{ }_{i+1 / 2}=\max \left(0,(\rho v)^{*}{ }_{i+\frac{1}{2}}\right)
\end{array}\right. \text { and }
$$

where $v$ and $\rho$ are the velocity and dislocation density of type $\alpha$ dislocations. When the dislocation velocity vector points from a free surface into the simulation space, there would be no influx, i.e. both the dislocation density and velocity on the upwind side would be zero, satisfying the no-entry boundary condition. Conversely when the dislocation velocity points from the inside of the simulation space to a free surface, the downwind dislocation density and dislocation velocity would be the same as that in the boundary voxel - such zero-streamwise gradient approximation is a commonly adopted convention as an exit boundary condition (Zikanov 2010).

In the simulation, the "left" and "right" hand sides correspond to the "upwind" and "downwind" sides of the dislocation flux. Therefore, for a type $\alpha$ dislocation density with a positive average velocity over the whole simulation space along the direction in which the flux is evaluated (i.e. either the $\mathrm{x}-, \mathrm{y}$ - or $\mathrm{z}$ - direction), the left state would be the $(i-1)$ side, while the left state for negative average velocity along the axis would be the $(i+1)$ side. 


\subsection{Dislocation generation, annihilation and cross slip}

In the edge-screw idealization scheme shown in Fig. 1, as discussed by Arsenlis et al. (2004), new lengths of edge dislocations are generated whenever a high gradient of flux of screw dislocations exists, and vice versa. The main idea is illustrated in Fig. 3(a), which shows that if edge dislocations in adjacent voxels do not glide with similar fluxes, a new density of screw dislocations will be generated to connect the two densities in the two voxels at their interface, and vice versa. To implement this idea, the generation algorithm used is as follows:

$$
\begin{gathered}
\dot{\rho}_{s+}^{i, i+n}=\left[\left(\vec{n}_{w} \cdot \vec{\xi}\right) /\left(\left|\vec{n}_{w}\right||\vec{\xi}|\right)\right]\left[F\left(\left(\frac{\rho v}{\Delta x}\right)_{e+}^{i+n}-\left(\frac{\rho v}{\Delta x}\right)_{e+}^{i}\right)+F\left(\left(\frac{\rho v}{\Delta x}\right)_{e-}^{i+n}-\left(\frac{\rho v}{\Delta x}\right)_{e-}^{i}\right)\right] \\
\dot{\rho}_{s-}^{i, i+n}=\left[\left(\vec{n}_{w} \cdot \vec{\xi}\right) /\left(\left|\vec{n}_{w}\right||\vec{\xi}|\right)\right] \cdot\left[F\left(\left(\frac{\rho v}{\Delta x}\right)_{e+}^{i}-\left(\frac{\rho v}{\Delta x}\right)_{e+}^{i+n}\right)+F\left(\left(\frac{\rho v}{\Delta x}\right)_{e-}^{i}-\left(\frac{\rho v}{\Delta x}\right)_{e-}^{i+n}\right)\right], \\
\text { etc., }
\end{gathered}
$$

where $i$ and $(i+n)$ are two neighboring voxels, and $F(x)$ is the ramp function (i.e. $F(x)=x$ if $x>0$, and 0 otherwise). The generated densities are partitioned back into the two neighboring voxels equally. The factor $\left(\vec{n}_{w} \cdot \vec{\xi}\right) /\left(\left|\vec{n}_{w}\right||\vec{\xi}|\right)$ here, where $\vec{n}_{w}$ is the unit vector normal to the wall between the two voxels, ensures that those dislocation densities with glide directions perpendicular to the voxel wall are not counted since no trailing dislocations can be produced along the voxel wall due to their movement. In addition, the total line energy of the new dislocations generated must be upper-bounded by the work done of the net effective stress. Since the power density for a given slip system $\alpha$ is $\rho_{\alpha} v_{\alpha} b \tau_{\alpha}^{e f f}$, the generation rates in eqn. (13) are scaled as follows:

$$
\begin{gathered}
\dot{\rho}_{s+}^{i, i+n} \rightarrow \frac{\dot{\rho}_{s+}^{i, i+n}}{E_{s}\left(\sum_{n} \dot{\rho}_{s+}^{i, i+n}\right)} \times \rho_{s+} v_{s+} b \tau_{s+}^{\text {eff }} ; \\
\dot{\rho}_{s-}^{i, i+n} \rightarrow \frac{\dot{\rho}_{s-}^{i, i+n}}{E_{S}\left(\sum_{n} \dot{\rho}_{s-}^{i, i+n}\right)} \times \rho_{s-} v_{s-} b \tau_{s-}^{e f f} ; \quad \text { etc. }
\end{gathered}
$$

where $E_{s}$ is the energy per unit length of dislocation.

Following Arsenlis et al. (2004), annihilation of dislocations occurs whenever dislocation densities of opposite signs co-exist within a critical capture radius. The annihilation rates used are:

$$
\begin{aligned}
& \dot{\rho}_{e+}^{a n n}=\dot{\rho}_{e-}^{a n n}=-\rho_{e+} \rho_{e-} R_{e}\left|v_{e+}-v_{e-}\right| \\
& \dot{\rho}_{s+}^{a n n}=\dot{\rho}_{s-}^{a n n}=-\rho_{s+} \rho_{s-} R_{s}\left|v_{s+}-v_{s-}\right|
\end{aligned}
$$

where $R_{e}$ and $R_{S}$ are the critical radii for edge and screw characters respectively within which annihilation would occur (Figure 3(c)). 
Cross slip for screw dislocations is also allowed in the present simulations with probability $P$ given as (Madec et al. 2002):

$$
P=\mathrm{e}^{\left(-\frac{E_{A}}{k T}\right)}
$$

where $k$ is the Boltzmann constant, $T$ is the absolute temperature, and the activation energy $E_{A}$ is assumed to be $0.5 \mathrm{eV}$.

\subsection{Dimensional analysis}

Since this work aims at providing a general model for a prototypical material, parameters for specific materials are not used but instead, the parameters are normalized. In the formulae below, normalized parameters are denoted as $(\stackrel{\sim}{*})$ while their real counterparts are denoted as $(\cdots)$.

First, all stress quantities are normalized by the shear modulus $\mu$, i.e.

$$
\tilde{\tau}=\tau / \mu
$$

and all lengths are normalized by the Burgers vector $b$, i.e.

$$
\tilde{x}=x / b
$$

The conversion between normalized dislocation density $\tilde{\rho}$ and the real density $\rho$ is therefore

$$
\tilde{\rho}=\rho b^{2}
$$

The normalized form of the velocity law used in the simulations is $\tilde{v}=\tilde{\tau}^{m}$, and so from eqns. (6) and (17) the normalized velocity $\tilde{v}$ is related to the real velocity $v$ via

$$
\tilde{v}=\frac{v}{v_{0}}\left(\frac{\tau_{0}}{\mu}\right)^{m}
$$

The normalized time is derived by dividing the normalized distance in eqn. (18) by the normalized velocity in eqn. (19), giving

$$
\Delta \tilde{t}=\frac{v_{0}}{b}\left(\frac{\mu}{\tau_{0}}\right)^{m} \Delta t
$$


Since time is normalized as in the above equation while strain is dimensionless, the strain rate is normalized as:

$$
\tilde{\varepsilon}=\frac{b}{v_{0}}\left(\frac{\tau_{0}}{\mu}\right)^{m} \dot{\varepsilon}
$$

With known material constants $\mu, b$ and $v_{0} / \tau_{0}{ }^{m}$, the simulated relationships between stress, strain and time, etc., can be related to the real parameters. In the present simulations, $m$ was set to be 1 .

\section{Simulation Details and Initial Dislocation Density Distributions}

Single crystals of various sizes were loaded at a constant nominal strain rate of $2.5 \times$ $10^{-3} s^{-1}$ which is a representative experimental value. The crystals were oriented in the cubic orientation with respect to the loading axis, namely, the tensile direction which was the $z$ direction was applied along [001]. The surfaces normal to the $x$ - and $y$-axes were set to be free surfaces, while periodic boundary conditions were applied along the z-axis, so that the specimen can be thought of as a wire of infinite length along the z-axis.

The RVE or voxel size was $(100 b, 100 b, 100 b)$ for the smallest specimen of size $(1000 b, 1000 b, 1000 b)$, and was fixed as $(200 b, 200 b, 200 b)$ for other specimen sizes of $(2000 b, 2000 b, 1000 b),(4000 b, 4000 b, 1000 b),(6000 b, 6000 b, 1000 b),(8000 b, 8000 b, 1000 b)$ and $(16000 b, 16000 b, 1000 b)$. In the simulation, the dislocation speed was capped at $20 \mu \mathrm{m} / \mathrm{s}$. In the following, normalized material properties are presented in general, but when the context requires real properties, these were calculated from their normalized counterparts by the following assumed material parameters: $b=2.863 \times 10^{-10} \mathrm{~m}, \mu=26 \times 10^{9} \mathrm{~Pa}, \tau_{0}=7.8 \times 10^{6} \mathrm{~Pa}$ and $v_{0}=1 \mathrm{~ms}^{-1}$.

In our previous study (Leung et al. 2015), the initial density of each of the 48 dislocation types in each simulation voxel was generated from a Gaussian distribution, giving rise to an overall, average dislocation density of $10^{12} \mathrm{~m}^{-2}$. While this serves as a convenient simulation scheme, this may not represent the realistic experimental situations, where micro-pillars are usually milled from a bulk crystal with a given microstructure (Uchic et al. 2004; Greer et al. 2005, Kiener et al. 2008; Ng and Ngan 2008b; Kim et al. 2009). For this reason, we employed another type of initial dislocation distribution in this work, which is a more discrete distribution with some voxels containing particularly high dislocation densities separated by relatively large areas of low dislocation densities, as shown in Figure 4, with the spatially averaged dislocation density maintained at $10^{12} \mathrm{~m}^{-2}$. This microstructure was used as a master microstructure from 
which specimens of different smaller sizes were cropped and simulated. In this scheme, different specimens of the same size will have different initial distributions of dislocation density, and this mimics the experimental procedure described above, in which micro- or nano-specimens milled out from a bulk crystal are mechanically tested to yield an ensemble behavior for a particular specimen size (Ngan and $\mathrm{Ng}, 2010$ ). The typical microstructure of a realistic bulk crystal contains entanglements of dislocations separated by low dislocation-density regions, with a typical separation on the order of microns between the dislocation entanglements. Thus, for the small voxel size considered in this study (e.g. $200 \mathrm{~b}$, i.e. $\sim 57.3 \mathrm{~nm}$ ), the initial dislocation density distribution should likely be rather discrete than continuous, and should represent better the real microstructure of a small specimen. Figure 5(a-c) show the percentage of voxels versus different initial dislocation densities for specimens of size $1000 \mathrm{~b}, 4000 \mathrm{~b}$ and $16000 \mathrm{~b}$ sampled from the master structure in Figure 4, and it can be seen that discreteness occurs in the initial dislocation density distribution in all the cases. In particular, total dislocation densities $\geq 4 \times$ $10^{12} \mathrm{~m}^{-2}$ are present for the larger specimens, and in the following, such high densities of dislocations are referred to as "clusters".

To produce the master microstructure shown in Figure 4, a specimen of dimensions $16000 b \times 16000 b \times 1000 b$, with periodic boundary conditions at all boundaries, was loaded with a high strain rate of $5 \times 10^{-2} s^{-1}$, until the dislocation pattern showed a high degree of nonuniformity, with clusters of dislocations separated by low-density regions. To ensure that the dislocation separations are comparable to that of as-annealed specimens, this structure was scaled up by 5 times to $80000 b \times 80000 b \times 5000 b$, i.e. $400 \times 400 \times 25$ voxels, so that the dislocation cluster separations became $\sim 1$ to $2 \mu \mathrm{m}$. Then, the dislocation density of the whole

specimen was re-scaled to $1 \times 10^{12} \mathrm{~m}^{-2}$ to produce the master microstructure shown in Figure 4. As said above, smaller specimens of sizes $1000 b \times 1000 b \times 1000 b, 2000 b \times 2000 b \times$ $1000 b, 4000 b \times 4000 b \times 1000 b, 8000 b \times 8000 b \times 1000 b$ and $16000 b \times 16000 b \times 1000 b$, were cropped from this master microstructure, to mimic an experimental process of milling small specimens out from a bulk crystal.

\section{Simulation Results}

\subsection{Size dependence of strength}

Figure 6 shows the simulated stress-strain curves of specimens of various sizes. It can be seen that the tensile strength increases with decreasing specimen size. Also, the smaller specimens exhibit an obvious initial yield point, and this diminishes for the larger specimens. Figure 7 plots the resolved shear strengths converted from the simulated initial yield points, together with typical experimental data and calculated yield stresses for different fcc metals. It can be seen that the present results are in broad agreement with known reported data. In particular, the approximate power-law size dependence of strength is well captured, and the 
present results are also similar to the DDFD simulated results in our previous study with a Gaussian initial dislocation-density distribution (Leung et al. 2015), indicating that the powerlaw trend between strength and size is robust with respect to the choice of the initial dislocation density distribution. Figure 6 further shows that after the initial yield, the flow stress fluctuates about a steady value for all specimen sizes simulated. Figure 8 shows the log-log plots of $0.2 \%$ proof stress versus the specimen size. The variation between the $0.2 \%$ flow stress and size also roughly follows the power-law trend $\sigma \sim D^{-m}$, with the power-law exponent $m$ roughly averaging to 0.416 . However, the data in Figure 8 in fact exhibit some bi-linearity as indicated by the dotted guiding line. In particular, the bi-linearity is caused by the simulation sets with no strong initial dislocation clusters for the $4000 \mathrm{~b}$ and $8000 \mathrm{~b}$ cases - if these two cases are omitted, the $\log \sigma$ vs $\log D$ trend would look quite linear as shown by the green symbols. The small scatter in $0.2 \%$ proof stress for the two smallest specimen sizes suggests that the initial microstructure did not affect the strength much.

\subsection{Dislocation accumulation during deformation}

Figure 6 shows that a sharp initial yield point occurs for specimens $<4000 \mathrm{~b}$ but not for larger specimens, and Figure 8 shows bilinearity of the simulation data. Both phenomena suggest that two different mechanisms are at play for the $<4000 \mathrm{~b}$ regime and the $\geq 4000 \mathrm{~b}$ regime. To illustrate these two mechanisms from a dislocation storage point of view, Figure 9 shows the total dislocation density evolution during deformation for different specimen sizes. It can be seen that the smaller specimens first undergo a considerable drop in dislocation density, and eventually end up with an almost constant dislocation density of around 10-50\% higher than the initial value, whereas the larger specimens undergo an insignificant change in the dislocation density initially, followed by a subsequent gradual build-up. By noting that strain is proportional to time at constant strain-rate, the stress peaks in Figure 6 for the smaller specimens correspond to the dislocation starvation condition in Figure 9 (Greer and Nix 2006, Shan et al. 2008). Figure 9 also shows that the dislocation density exhibits gradual build-up for specimen sizes $>4000 b$, i.e. $\sim 1.15 \mu \mathrm{m}$, and remains roughly constant for specimen sizes for specimen sizes $<4000 b$. Figure 9 is generally indicative of an increase in dislocation storage capability in larger specimens. This is probably because the dislocation depletion rate in specimens larger than $4000 \mathrm{~b}$ or so is relatively low due to the low surface-area to volume ratio, so that a high proportion of the generated dislocation density is retained within the specimen.

Figure 10 plots the relationship between the spatially averaged dislocation generation rate and spatially averaged rate of loss of dislocation density to the free surfaces. For the smaller specimens, both the generation and loss rates are high, but these two rates roughly cancel out each other so that the data fall on the $45^{\circ}$ line. Since the dipole annihilation rate was usually 1 to 2 orders of magnitude smaller than the generation rate, if the generation rate roughly equals the loss rate, the specimen would undergo almost no change in overall dislocation density, which 
agrees well with the observation from Figure 9 for specimens $<4000 \mathrm{~b}$. For the larger specimens, however, the generation rate outweighs the loss rate, as shown by the data points in Figure 10 which fall above the $45^{\circ}$ line for specimen sizes $>4000 \mathrm{~b}$, corresponding to the gradual increase in dislocation density during loading in Figure 9. Figure 10 also shows that the normalized yield stress is linearly related to the average loss rate due to flux out of the specimen for different specimen sizes. This is probably because the spatially averaged loss rate is linearly related to the spatially averaged generation rate, and in turn the dislocation generation scales with the external work done by applied stress (see section 2.3), accounting for the linear relationship between the density loss rate and proof stress. Figure 10 indicates that the smaller specimens with a larger surface-area to volume ratio exhibit a higher loss rate of dislocations, which has to be balanced by a higher generation rate, and this would in turn require a higher applied stress. As for specimen sizes $\geq 4000 \mathrm{~b}$, this effect is still at play, but the bi-linearity of the plot in Figure 8 suggests that this starvation effect is gradually diminishing in the larger specimen range, thus a different mechanism should control the size effect in the $\geq 4000 \mathrm{~b}$ regime. This additional mechanism will be discussed later.

\subsection{Spatial distributions of dislocation-density, strain and generation during deformation}

To gain further insight into the mechanisms controlling the size effect of strength, in Figure 11(a-c) are plotted the simulated spatial evolutions of dislocation density, strain and generation as specimens of different sizes undergo deformation. Figure 11(a) shows the dislocation-density contour maps for several specimen sizes at different strain levels. While the dislocations are concentrated in the core region for the smaller specimens, for larger specimens, clusters of dislocations are scattered around the whole specimen, with a relatively lower density at the free surfaces. The clustered cases correspond well to the so-called "soft-surface, hard-core" structure commonly observed in experiments (Fourie 1970), in which the observed dislocation density is higher in the core region. In addition, the low dislocation density in the proximity of free surfaces indicates that free surfaces are effective sinks for dislocations. Thus, in the initial stage of deformation where the dislocation generation rate is low, the rate of dislocation escape is the highest in the smallest specimen due to the highest surface area to volume ratio.

In the strain contour plots in Figure 11(b), the smallest $1000 \mathrm{~b}$ specimen has the higher strain in the core region of specimen, indicating that the core region is softer than the surface region, whereas strain is more uniform in the $16000 \mathrm{~b}$ case. For the $4000 \mathrm{~b}$ case selected, the specimen contained some high-density clusters in the initial distribution, and on deformation, the strain remains low in these clustered regions, even though they are situated in the supposedly soft core region. The dense dislocation clusters pre-existing in the specimen are associated with high Taylor hardening during deformation, so that they are virtually immobile with low local strains. In addition, it can be observed from Figure 11(a,b) that regions with an initial dislocation density between $\sim 1 \times 10^{12} \mathrm{~m}^{-2}$ to $1.5 \times 10^{12} \mathrm{~m}^{-2}$ are softest with the highest cumulative strains. 
The dislocation multiplication behavior in the specimens can also be illustrated by the cumulative generation pattern plots shown in Figure 11(c), which represents the cumulative density of dislocations generated through the thickness of specimens on the (111) plane. The smallest $1000 \mathrm{~b}$ specimen has most of the dislocations generated in the core region, whereas the largest $16000 \mathrm{~b}$ specimen exhibits more uniform generation patterns, in which certain parts of the core region are relatively inactive, probably because dense dislocation clusters are present there which suppress dislocation fluxes. For the $4000 \mathrm{~b}$ specimen, the high-density clustered core region as well as the lower left corner of the specimen show lower generation levels. Recalling from section 2.3 that dislocation generation arises from flux differences between adjacent voxels, and since flux differences would likely scale up or down with the local fluxes, dislocation generation would be rather inactive in regions with dense dislocation clusters which are immobile. In fact the similarity between Figures 11(b) and 11(c) suggests certain correlation between the ease of deformation and the local multiplication behavior.

\section{Discussion}

\subsection{Key findings from the simulations}

The key findings from the present simulations are summarized as follows:

(i) Both the simulated yield strength and the $0.2 \%$ proof stress roughly follow the "smaller being stronger" size effect (Figures 6 to 8), in good agreement with the typical power-law trend $\sigma \sim D^{-m}$ for a considerably large size range, from $1000 b$ to $16000 b$ (Figure 7).

(ii) Notwithstanding (i) above, a slight bi-linearity of the $\log \sigma$ vs $\log D$ plot is observed in Figure 8, with the turning point at the specimen size of $\sim 4000 \mathrm{~b}$.

(iii) During deformation, specimens smaller than $\sim 4000 b$ do not exhibit dislocation accumulation (Figure 9) due to cancellation of the dislocation generation rate by the loss rate from the specimen (Figure 10). For specimens larger than $\sim 4000 b$, rapid dislocation accumulation happens during deformation (Figure 9), due to a higher generation rate than the loss rate (Figure 10).

(iv) For very small specimens in the $<4000 \mathrm{~b}$ regime, dislocation generation is concentrated in the core region of the specimen, which is also the soft part of the sample with the highest strain. For larger specimens in the $>4000 \mathrm{~b}$ regime, they more likely contain regions with dense dislocation clusters in the initial microstructure which are immobile with very low strain and dislocation generation (Figure 11).

(ii) to (iv) above suggest two different mechanisms that control strength for specimen sizes below $\sim 4000 \mathrm{~b}$ and above. Close inspection of Figure 7 shows that a bi-linearity in the $\log$ $\sigma$ vs $\log D$ plot may not be obvious for most of the experimental data set shown, due to the large 
scatter typical for this type of experiments. However, the Ni data by Schneider et al. (2013), the green diamond symbols in Figure 7, indeed exhibit a rather obvious bi-linear behavior, with the turning point at $\sim 4000 \mathrm{~b}$, which agrees well with the present prediction. Furthermore, the Au data set by Volkert and Lilleodden (2006), the blue diamond symbols, also shows a weak but discernible bi-linear trend with a turning point at a similar specimen size. In fact, it is impossible for the power-law trend $\sigma \sim D^{-m}$ to continue for indefinitely increasing specimen size, since then the strength would be zero. The strength-size relation should certainly deviate from the powerlaw trend for the small size regime, and so the present observed bi-linear behavior is not unreasonable.

The two mechanisms that control strength in the $<4000 \mathrm{~b}$ regime and above are discussed in the following.

\subsection{Dislocation starvation mechanism}

For the size range of $<4000 b$, the mechanism governing the size effect is evidently dislocation starvation (Greer and Nix 2006, Shan et al. 2008). In this regime, the stress-strain curve shows a characteristic sharp initial yield point (Figure 6) arising from an initial depletion of dislocations, followed by a roughly constant level of overall dislocation density (Figure 9). No formation or retention of dislocation clusters was observed (Figure 11(a)). As discussed in section 4.2, the spatially averaged dislocation loss rate increases with decreasing specimen sizes, due to the increasing surface area-to-volume ratio, as well as the effective sinking nature of the free surface. According to the Orowan equation in eqn. (9), in order for the specimen to deform at the prescribed constant strain rate, continuous dislocation multiplication is required to balance the density loss, mainly due to dislocations zipping out of the small specimen. Since the dislocation generation considered in the present simulation scheme is upper-bounded by the work done by external stress, smaller specimens require a higher applied stress for more rapid generation. It is interesting to see from Figures 6 and 9 that for specimens larger than $4000 b$, although there is net dislocation accumulation, there is still a slight reduction in flow stress as the specimen size increases, and the stress-strain response exhibits no obvious strain hardening. This indicates that strength in this size and dislocation-density regime studied is still affected significantly by the Orowan equation, in addition to the Taylor hardening. As more mobile dislocations are accumulated in a larger specimen, there will be a softening effect due to the Orowan equation, but the fact that the flow stress does not rise indicates that such a softening effect more or less balances the increased Taylor hardening effect. It should be noted that in our recent work (Leung et al., 2015), periodic boundary conditions were used so that dislocation accumulation was much more rapid inside the simulation cell, and significant strain hardening in the stress-strain response was predicted. 


\subsection{Weakest Link regime}

For specimen sizes $>4000 \mathrm{~b}$, the mechanism governing strength is related to the initial dislocation microstructure, as is similar to the weakest-link concept (El-Awady et al. 2009). In this regime, only small or even no stress peaks arise in the stress-strain behavior (Figure 6), and as deformation continues, initial depletion of dislocations is insignificant and growth afterwards is persistent (Figure 9). Moreover, dislocation clusters form easily during deformation, and any pre-existing dense clusters are likely preserved during deformation. For instance, the dense cluster in the top right corner of the $16000 \mathrm{~b}$ specimen in Figure 11(a) remains during straining although this is close to a free surface, due to low mobility of dislocations there as a result of the high Taylor hardening.

Figure 8 indicates that the initial microstructure is important to the size effect. As discussed in section 4.3, pre-existing dense dislocation clusters in the initial microstructure tend to remain as undeformable regions throughout the deformation (Figure 11(a,b)), because of the high Taylor hardening stress and low dislocation multiplication rate there. In addition, from Figure 11, the neighborhood of a dense clustered region is also prone to be less deformable. This is evident from the $4000 \mathrm{~b}$ specimen in Figure 11 which has the lowest dislocation density, strain level as well as multiplication level in the lower left corner of the specimen. To see how the presence of dense clusters would affect their neighborhood, Figure 12(a,b) show the enlarged views of Figure 11(a,b) respectively. The red contours in Figure 12(a,b) outline a soft region, with a moderate density level between $\sim 1 \times 10^{12} \mathrm{~m}^{-2}$ to $1.5 \times 10^{12} \mathrm{~m}^{-2}$ described in section 4.3 and a high strain of $\sim 0.2 \%$, in the proximity of some dense clusters. On the other hand, the green contours outline a few hard regions with low density and strain levels, closely surrounded by dense dislocation clusters. In Figure 12, the red-outlined region exhibits the optimal dislocation density level for easiest deformation described in section 4.3, and the material points inside are typically situated at distances $2000 \mathrm{~b}$ to $3000 \mathrm{~b}$ away from any dense clusters. For locations within a distance of $\sim 2000 \mathrm{~b}$ from dense clusters, the mobile dislocation density is prone to be low, and such a region is likely a hard region, as exemplified by the green-outlined regions in Figure 12. Since specimen sizes of $4000 \mathrm{~b}$ and $8000 \mathrm{~b}$ are just barely larger than the critical length scale of $2000 \mathrm{~b}$ to $3000 \mathrm{~b}$ here, truncation of the volume fraction of "soft" regions is more significant than in yet larger specimens (e.g. 16000b). The neighborhood of dense clusters is harder since the dense clusters block dislocations from reaching there. This suppresses dislocation density growth, and so the lack of dislocations as agents for plasticity (c.f. the Orowan equation) results in hardening. As a result, a smaller sample is harder in this size regime. It is thus apparent that the presence of dislocation clusters would affect the volume fraction of soft regions, which determines strength in this size regime according to the weakest-link concept (El-Awady et al. 2009).

The critical length scale $2 r$ here is obviously related to the mean size of the soft regions in the master microstructure in Figure 4. To see this, the density map in Figure 4(a) is converted 
into a bitmap as shown in Figure 4(b), using a cutoff density at the average value of $1 \times$ $10^{12} \mathrm{~m}^{-2}$. With such a cutoff density, the initial microstructure becomes one consisting of lowdensity regions separated by high-density regions resembling a cellular structure. Figure 4(c) shows the corresponding "hole-counting" analysis (Zaiser et al. 1999) by plotting the cumulative number of cells of size $\lambda$ greater than $\Lambda$ versus $\Lambda$ in double-logarithmic scale. The cell size values $\lambda$ were determined by the line-intercept method, with 20 vertical and 20 horizontal measurement lines. From Figure 4(c), the initial cell size distribution is actually far from a fractal, with the majority of cell sizes ranging from $3000 \mathrm{~b}$ to $10000 \mathrm{~b}$. Hence, a mean cell size can be obtained

as $\bar{\lambda}=\int_{0}^{\infty} \lambda p(\lambda) d \lambda \sim 4200 b$, where $p(\lambda)$ is the probability density of $\lambda$. This value of $\bar{\lambda}$ is of a similar magnitude as the critical length scale $2 r$ discussed above concerning Figure 12.

Table 1 shows the percentage of "soft" voxels, for which $\varepsilon_{\text {voxel }}>70 \% \times \varepsilon_{\text {total }}$, in different specimen sizes and initial dislocation densities. From the table, the $1000 \mathrm{~b}$ and $2000 \mathrm{~b}$ specimens are only slightly affected by the initial microstructure. For specimen sizes $\geq 4000 \mathrm{~b}$, a lower percentage of soft voxels is usually resulted for higher initial dislocation density for the same specimen size. In addition, when there are pre-existing dense dislocation clusters (i.e. the cases marked by asterisks in Table 1), increasing the specimen size from $4000 \mathrm{~b}$ to $16000 \mathrm{~b}$ would lead to an increase in the percentage of soft voxels and hence a lower overall strength. The reason is that as the specimen size increases, deformation in the specimen becomes more uniform and is not mainly confined to the core region, as evident from Figure 11(b). In addition, as the specimen size increases, the initial dislocation density should converge to $1 \times 10^{12} \mathrm{~m}^{-2}$, so that situations with an unusually high initial dislocation density should be increasingly rare. Considering that a "dense dislocation cluster" discussed so far refers to regions with local density $\geq 4 \times 10^{12} \mathrm{~m}^{-2}$, it is quite likely that in real experiments, such clusters are ample in micron sized specimens, with a separation of the order of $\sim 1$ to $2 \mu \mathrm{m}$, and so the weakest-link mechanism discussed in this section should be at play.

\subsection{Scatter in $0.2 \%$ proof stress}

The typical scatter in the yield stresses for specimens cropped from the master structure in Figure 4 is presented in Figure 13. The two smallest specimen sizes do not show much dependence of strength on the initial dislocation density, while for the three cases with larger specimen sizes the initial dislocation density exerts a stronger effect on the yield stress. The lack of correlation between the initial dislocation density and yield stress in the smaller specimens is due to the fact that the initial dislocation structure cannot persist throughout the loading process, due to the higher stress level attained by the smaller specimens. Thus, any dislocation clusters initially present would disentangle and disappear from the specimen during the loading process, so that the initial dislocation density does not significantly affect the yield stress in small specimens. As for the $4000 b-16000 b$ specimens, Figure 11(a) shows that the initial dense 
clusters more or less remain within the sample throughout the loading process. A higher initial dislocation density therefore means more dense clusters retained, and this accounts for the positive correlation between the initial dislocation density and strength. It can be expected that when the specimen size increases further and exceeds the internal length scale, namely, the mean separation between the clusters of dislocations in the master microstructure, the initial dislocation distribution in samples randomly cropped from the master microstructure should converge to that of the latter, so that the scatter in the yield stress should eventually diminish.

\subsection{Directions for future development}

With the present average dislocation density and voxel size, it is possible that a crosssection of a voxel may contain less than one dislocation on average, i.e. $\rho(\Delta x)^{2}<1$ where $\Delta x$ is the voxel length. However, since dislocations may not always exist as straight lines running through the voxel length but sometimes as small segments in loops, such low densities encountered in the coarse-graining concept involved would still mean that some dislocation content is present. For micro-sized volumes containing an average dislocation density on the order of $10^{12} \mathrm{~m}^{-2}$, one scenario would be a dilute-discrete picture in which only a few continuous dislocation segments are present. In a density representation of such a dilute-discrete picture, certain voxels can be made to contain much higher density values representing the discrete dislocations, while the rest of the voxels can contain vanishing density values representing the free spaces between the discrete dislocations. In the present simulations, the initial dislocation distributions were chosen to be not of such a dilute-discrete nature, but instead, rather smooth density distributions were used which would represent a scenario with very small defect loops or segments continuously distributed around. Such a picture would be close to, for example, a state after FIB fabrication, although it was not our specific purpose here to look at the effects of FIB machining. The dilute-discrete case can be studied as future work.

In typical load-controlled tests, the stress-strain curves would show constantly increasing stress (Ng and Ngan 2008b), and in strain rate or displacement rate controlled tests (Uchic et al. 2004, Kim et al. 2009, Kim et al. 2012) the stress typically fluctuates about a steady value which does not differ too much from the initial yield stress. In Figure 6, however, the simulated stressstrain curves show a peak in stress (i.e. the yield stress) in the initial loading stage, followed by small fluctuations about a considerably lower stress value, resembling the results of Brenner (1957). As discussed above, the stress drop after the initial yield in the present simulations is due to the triggering of rapid dislocation multiplication activity that relieves the initial "dislocation starvation" problem. While in real cases, dislocations may simply zip out of the specimen without contributing to dislocation multiplication, in the present simulation scheme (c.f. eqns. (13)), dislocation fluxes arising from dislocation glide may over-generate dislocations. Although generation is already upper-bounded by the work done by the external applied stress (c.f. eqn. (14)), no energy loss is assumed. In addition, the dislocation flux gradients may have 
been over-estimated, since the line tension effect of the connecting dislocations across two neighboring voxels in reality would reduce the difference in the dislocation flux between the two voxels. Yet, this line tension effect is complicated and has not yet been dealt with in the present work. Another possible reason is that the stress-control of the current simulation is still not sophisticated enough to tackle over-shoots in stresses. Further work on improving the DDFD simulation protocol along these directions would be of great significance.

Finally, the present simulation results in Figures 6 to 8 indicate a strong size dependence of the yield strength which is in reasonable agreement with experimental data as Figure 7 shows. This is a remarkable result, given that the present simulator is based only on dislocation density functions without discrete, individual dislocation lines considered. Unlike Parthasarathy et al. (2007) who showed that the truncation of dislocation sources would result in single-armed sources with length truncated by the specimen dimension, such size-dependent sources are ignored in the present simulation scheme. A more complete understanding of the size effect of deformation would require further efforts in incorporating the relevant discrete dislocation physics.

\section{Conclusion}

A new simulation methodology based on the dynamics of dislocation-density functions was employed in this work to study the tensile behavior of single crystal wires. The typical power-law "smaller being stronger" size effect was predicted in micron- and sub-micron crystals. The simulation results nevertheless indicate the existence of two separate regimes for the size effect: a dislocation starvation regime for specimen sizes $<4000 b$, and a microstructure dominating regime for specimen sizes $>4000 \mathrm{~b}$. Essentially, the dislocation loss rate in the starvation regime is too high, so that a higher stress is required to provide the work done required for compensating rapid dislocation generation to sustain the deformation. As for the microstructure dominating regime, the size effect is found to be due to a mechanism akin to the weakest-link theory. For specimens $\geq 4000 \mathrm{~b}$, pre-existing dense dislocation clusters cannot easily disentangle, and their continued presence during deformation blocks dislocations from reaching their neighborhood, leading to reduced dislocation density and deformation there. It is found that locations farther than about $2000 \mathrm{~b}$ from dense dislocation clusters would exhibit an optimal dislocation density of $\sim 1 \times 10^{12} \mathrm{~m}^{-2}$ to $1.5 \times 10^{12} \mathrm{~m}^{-2}$ for deformation, and so would be particularly soft regions for deformation. A specimen size in the $4000 \mathrm{~b}$ to $8000 \mathrm{~b}$ range would not be large compared with the critical length scale of $\sim 2000 \mathrm{~b}$ here, and so the presence

of dense dislocation clusters in the initial microstructure would truncate off a larger fraction of the soft regions, thus resulting in higher strength, than larger specimens. 


\section{Acknowledgment}

The work reported here is supported by funding from Kingboard Endowed Professorship in Materials Engineering.

\section{References}

Acharya A 2001 A model of crystal plasticity based on the theory of continuously distributed dislocations Journal of the Mechanics and Physics of Solids 49 761-784

Akarapu S, Zbib H M and Bahr D F 2010 Analysis of heterogeneous deformation and dislocation dynamics in single crystal micropillars under compression International Journal of Plasticity $26239-57$

Alankar A, Mastorakos I N and Field D P 2009 A dislocation-density-based 3D crystal plasticity model for pure aluminum Acta Materialia 57 5936-46

Ananthakrishna G 2007 Current theoretical approaches to collective behavior of dislocations Physics Reports 440 113-259

Arsenlis A, Parks D M, Becker R and Bulatov V V 2004 On the Evolution of Crystallographic Dislocation Density in Non-homogeneously Deforming Crystals Journal of the Mechanics and Physics of Solids 52 1213-46

Asaro R J and Rice J R 1977 Strain localization in ductile single crystals Journal of the Mechanics and Physics of Solids 25 309-38

Balluffi R W 2012 Introduction to Elasticity Theory for Crystal Defects: Cambridge University Press)

Benzerga A A 2009 Micro-pillar plasticity: 2.5D mesoscopic simulations Journal of the Mechanics and Physics of Solids 57 1459-69

Brenner S S 1956 Tensile Strength of Whiskers Journal of Applied Physics 27 1484-91

Brenner S S 1957 Plastic Deformation of Copper and Silver Whiskers Journal of Applied Physics 28 1023-6

Busso E P, Meissonnier F T and O'Dowd N P 2000 Gradient-dependent deformation of twophase single crystals Journal of the Mechanics and Physics of Solids 48 2333-61

Chen Y S, Choi W, Papanikolaou S and Sethna J P 2010 Bending crystals: emergence of fractal dislocation structures Physical Review Letters 105105501

Chen Y S, Choi W, Papanikolaou S, Bierbaum M and Sethna J P 2013 Scaling theory of continmuum dislocation dynamics in three dimensions: self-organized fractal pattern formation International Journal of Plasticity 46 94-129.

Cheng B, Leung H S and Ngan A H W 2014 Strength of metals under vibrations - dislocationdensity-function dynamics simulations Philosophical Magazine 1-21

Choi W, Chen Y S, Papanikolaou S and Sethna J P 2012 Is dislocaton flow turbulent in deformed crystals? Computational Science and Engineering 14 33-39

Csikor F F, Motz C, Weygand D, Zaiser M and Zapperi S 2007 Dislocation avalanches, strain bursts, and the problem of plastic forming at the micrometer scale Science 318 251-4

De Hosson J T M, Soer W A, Minor A M, Shan Z, Stach E A, Syed Asif S A and Warren O L 2006 In situ TEM nanoindentation and dislocation-grain boundary interactions: a tribute to David Brandon Journal of Materials Science 41 7704-19 
Devincre B and Condat M 1992 Model Validation of a 3D simulation of dislocation dynamics: Discretization and Line Tension Effects Acta Metallurgica et Materialia 40 2629-37

Devincre B, Kubin L and Hoc T 2006 Physical analyses of crystal plasticity by DD simulations Scripta Materialia 54 741-6

Dou R and Derby B 2009 A universal scaling law for the strength of metal micropillars and nanowires Scripta Materialia 61 524-7

Dunne F P E, Rugg D and Walker A 2007 Lengthscale-dependent, elastically anisotropic, physically-based hcp crystal plasticity: Application to cold-dwell fatigue in Ti alloys International Journal of Plasticity 23 1061-83

El-Awady J A, Wen M and Ghoniem N M 2009 The role of the weakest-link mechanism in controlling the plasticity of micropillars Journal of the Mechanics and Physics of Solids $5732-50$

Fivel M C and Canova G R 1999 Developing rigorous boundary conditions to simulations of discrete dislocation dynamics Modelling and Simulation in Materials Science and Engineering 7 753-68

Fourie J T 1970 Sub-surface dislocation structure of deformed copper Philosophical Magazine 21 977-85

Franciosi P and Zaoui A 1982 Multislip in F.C.C. crystals: A theoretical approach compared with experimental data Acta Metallurgica 30 1627-1637

Frick C P, Clark B G, Orso S, Schneider A S and Arzt E 2008 Size effect on strength and strain hardening of small-scale [111] nickel compression pillars Materials Science and Engineering: A 489 319-29

Gómez-García D, Devincre B and Kubin L P 2006 Dislocation Patterns and the Similitude Principle: 2.5D Mesoscale Simulations Physical Review Letters 96125503

Greer J and Nix W 2006 Nanoscale gold pillars strengthened through dislocation starvation Physical Review B 73245410

Greer J R and De Hosson J T M 2011 Plasticity in small-sized metallic systems: Intrinsic versus extrinsic size effect Progress in Materials Science 56 654-724

Greer J R, Oliver W C and Nix W D 2005 Size dependence of mechanical properties of gold at the micron scale in the absence of strain gradients Acta Materialia 53 1821-30

Groma I, Csikor F F and Zaiser M 2003 Spatial correlations and higher-order gradient terms in a continuum description of dislocation dynamics Acta Materialia 51 1271-1281

Gu R and Ngan A H W 2013 Dislocation arrangement in small crystal volumes determines power-law size dependence of yield strength Journal of the Mechanics and Physics of Solids 61 1531-42

Hirschberger C B, Peerlings R H J, Brekelmans W A M and Geers M G D 2011 On the role of dislocation conservation in single-slip crystal plasticity Modelling and Simulations in Materials Science and Engineering 19085002

Hirth J P and Lothe J 1992 Theory of Dislocations, 2nd Ed. Krieger Publishing Co. 125

Huang M, Zhao L and Tong J 2012 Discrete dislocation dynamics modelling of mechanical deformation of nickel-based single crystal superalloys International Journal of Plasticity 28 141-58

Jennings A T, Burek M J and Greer J R 2010 Microstructure versus Size: Mechanical Properties of Electroplated Single Crystalline Cu Nanopillars Physical Review Letters 104135503

Jennings A T and Greer J R 2011 Tensile deformation of electroplated copper nanopillars Philosophical Magazine $\mathbf{9 1}$ 1108-20 
Kaufmann D, Mönig R, Volkert C A and Kraft O 2011 Size dependent mechanical behaviour of tantalum International Journal of Plasticity 27 470-8

Keller C, Hug E, Retoux R and Feaugas X 2010 TEM study of dislocation patterns in nearsurface and core regions of deformed nickel polycrystals with few grains across the cross section Mechanics of Materials 42 44-54

Kiener D, Grosinger W and Dehm G 2009 On the importance of sample compliance in uniaxial microtesting Scripta Materialia 60 148-51

Kiener D, Grosinger W, Dehm G and Pippan R 2008 A further step towards an understanding of size-dependent crystal plasticity: In situ tension experiments of miniaturized singlecrystal copper samples Acta Materialia 56 580-92

Kim J-Y and Greer J R 2009 Tensile and compressive behavior of gold and molybdenum single crystals at the nano-scale Acta Materialia 57 5245-53

Kim J-Y, Jang D and Greer J R 2009 Insight into the deformation behavior of niobium single crystals under uniaxial compression and tension at the nanoscale Scripta Materialia 61 300-3

Kim J-Y, Jang D and Greer J R 2012 Crystallographic orientation and size dependence of tension-compression asymmetry in molybdenum nano-pillars International Journal of Plasticity 28 46-52

Komanduri R, Chandrasekaran N and Raff L M 2001 Molecular dynamics (MD) simulation of uniaxial tension of some single-crystal cubic metals at nanolevel International Journal of Mechanical Sciences 43 2237-60

Lee S-W, Han S M and Nix W D 2009 Uniaxial compression of fcc Au nanopillars on an MgO substrate: The effects of prestraining and annealing Acta Materialia 57 4404-15

Lee S-W and Nix W D 2012 Size dependence of the yield strength of fcc and bcc metallic micropillars with diameters of a few micrometers Philosophical Magazine 92 1238-60

Legros M, Dehm G, Arzt E and Balk T J 2008 Observation of giant diffusivity along dislocation cores Science 319 1646-9

Leung H S, Leung P S S, Cheng B and Ngan A H W 2015 A new dislocation-density-function dynamics scheme for computational crystal plasticity by explicit consideration of dislocation elastic interactions International Journal of Plasticity 67 1-25

Limkumnerd S and Sethna J P 2006 Mesoscale theory of grains and cells: crystal plasticity and coarsening Physical Review Letters 96095503

Madec R, Devincre B and Kubin L P 2002 Simulation of Dislocation Patterns in Multislip Scripta Materialia 47 689-95

Motz C, Weygand D, Senger J and Gumbsch P 2009 Initial dislocation structures in 3-D discrete dislocation dynamics and their influence on microscale plasticity Acta Materialia 57 1744-54

Ng K S and Ngan A H W 2008a Breakdown of Schmid's law in micropillars Scripta Materialia $59796-9$

$\mathrm{Ng} \mathrm{K} \mathrm{S}$ and Ngan A H W 2008b Stochastic nature of plasticity of aluminum micro-pillars Acta Materialia 56 1712-20

Ngan A H W 2011 An explanation for the power-law scaling of size effect on strength in microspecimens Scripta Materialia 65 978-81

Oh S H, Legros M, Kiener D and Dehm G 2009 In situ observation of dislocation nucleation and escape in a submicrometre aluminium single crystal Nature materials 8 95-100 
Parrinello M and Rahman A 1981 Polymorphic transitions in single crystals: A new molecular dynamics method Journal of Applied Physics 52 7182-90

Parthasarathy T A, Rao S I, Dimiduk D M, Uchic M D and Trinkle D R 2007 Contribution to size effect of yield strength from the stochastics of dislocation source lengths in finite samples Scripta Materialia 56 313-6

Puri S, Das A and Acharya A 2011 Mechanical response of multicrystalline thin films in mesoscale field dislocation mechanics Journal of the Mechanics and Physics of Solids 59 2400-2417

Rezvanian O, Zikry M A and Rajendran A M 2007 Statistically stored, geometrically necessary and grain boundary dislocation densities: microstructural representation and modelling Proceedings of the Royal Society A: Mathematical, Physical and Engineering Sciences 463 2833-53

Roy A and Acharya A 2005 Finite element approximation of field dislocation mechanics Journal of the Mechanics and Physics of Solids 53 143-170

Schneider A S, Clark B G, Frick C P, Gruber P A and Arzt E 2009 Effect of orientation and loading rate on compression behavior of small-scale Mo pillars Materials Science and Engineering: A $\mathbf{5 0 8} 241-6$

Schneider A S, Kiener D, Yakacki C M, Maier H J, Gruber P A, Tamura N, Kunz M, Minor A M and Frick C P 2013 Influence of bulk pre-straining on the size effect in nickel compression pillars Materials Science and Engineering: A 559 147-58

Shan Z W, Mishra R K, Syed Asif S A, Warren O L and Minor A M 2008 Mechanical annealing and source-limited deformation in submicrometre-diameter Ni crystals Nature materials $7115-9$

Taupin V, Varadhan S, Fressengeas C and Beaudoin A J 2008 Directionality of yield point in strain-aged steels: the role of polar dislocations Acta Materialia 56 3002-3010.

Uchic M D, Dimiduk D M, Florando J N and Nix W D 2004 Sample dimensions influence strength and crystal plasticity Science 305 986-9

Van der Giessen E and Needleman A 1995 Discrete dislocation plasticity: a simple planar model Modelling and Simulation in Materials Science and Engineering 3 689-735

Volkert C A and Lilleodden E T 2006 Size effects in the deformation of sub-micron Au columns Philosophical Magazine $\mathbf{8 6}$ 5567-79

Wesseling P 2001 Principles of Computational Fluid Dynamics Springer-Verlag 381

Yamakov V, Wolf D, Phillpot S R, Mukherjee A K and Gleiter H 2002 Dislocation processes in the deformation of nanocrystalline aluminium by molecular-dynamics simulation Nature materials 1 45-8

Yefimov S and van der Giessen E 2005 Multiple slip in a strain-gradient plasticity model motivated by a statistical-mechanics description of dislocations International Journal of Solids and Strutures 42 3375-3394

Zaiser M, Bay K and Hähner P 1999 Fractal analysis of deformation-induced dislocation patterns Acta Materialia 47 2463-76

Zhou C, Biner S B and LeSar R 2010 Discrete dislocation dynamics simulations of plasticity at small scales Acta Materialia 58 1565-77

Zikanov O 2010 Essential Computational Fluid Dynamics. John Wiley \& Sons. p 29 
Figures

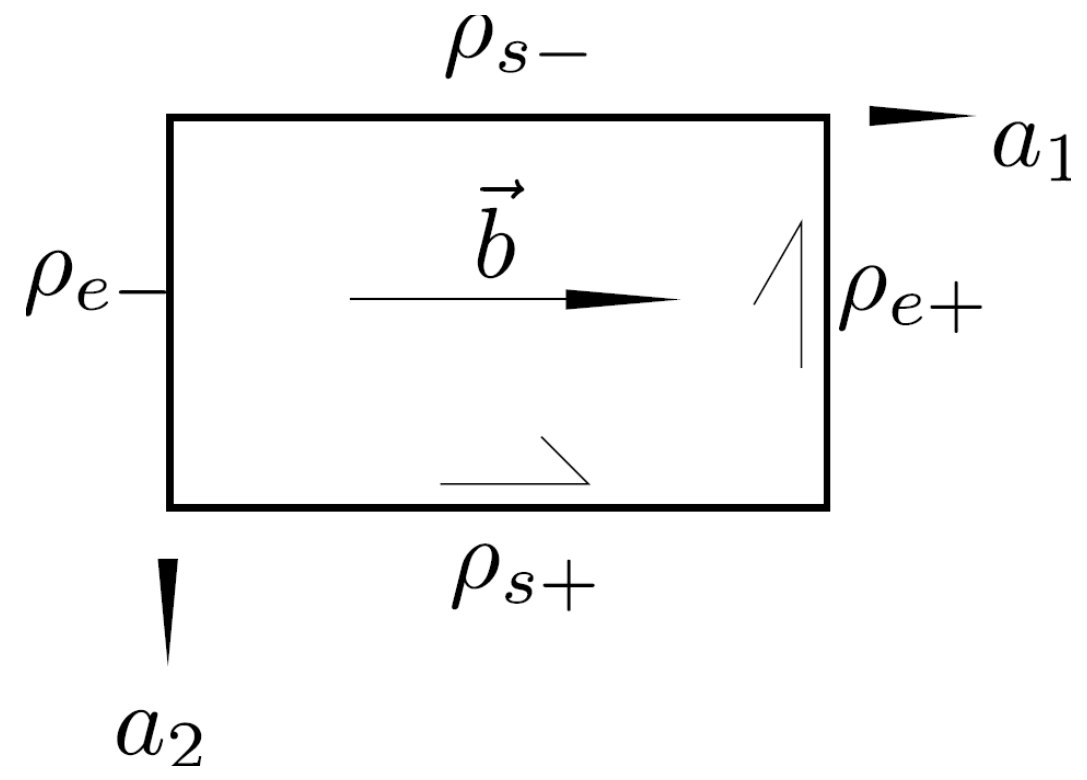

Figure 1 - Edge-screw idealization of slip systems.

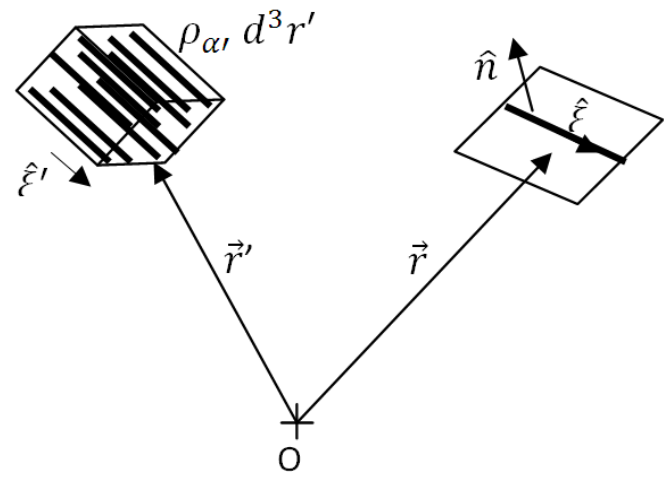

Figure 2 - Elastic interaction between a dislocation segment at $\vec{r}$ and a density of dislocations at $\vec{r}^{\prime}$. 

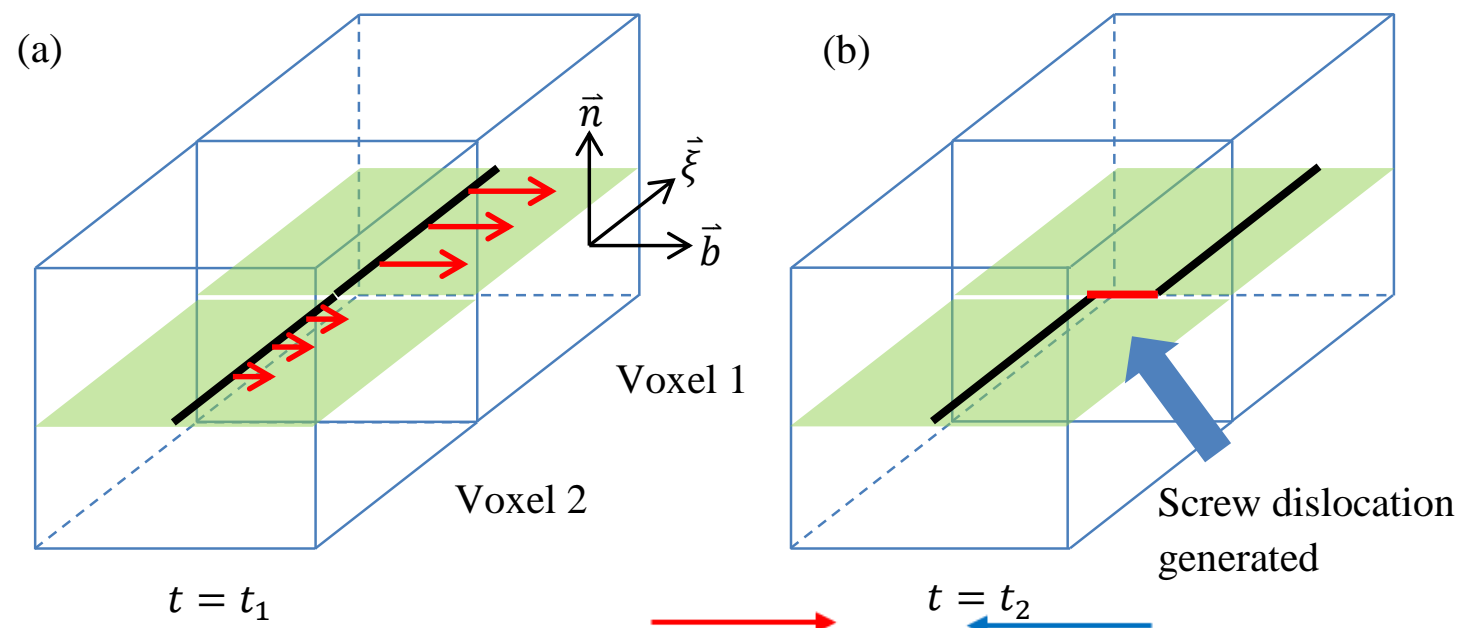

(c)

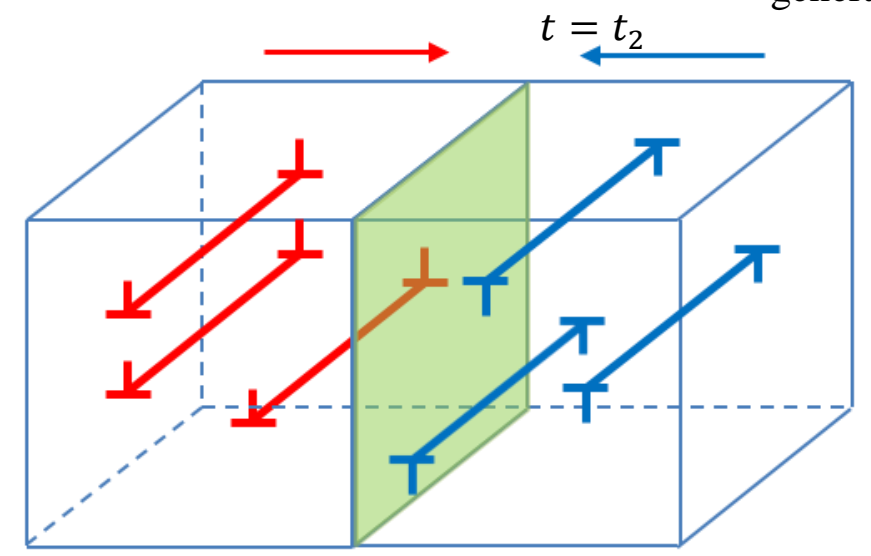

Figure 3 - (a) Edge dislocations of the same type glide at different speed in two neighboring voxels. (b) Production of a density of screw dislocation of the on the same slip plane and of the same burgers vector. (c) Dislocation annihilation in a voxel. 


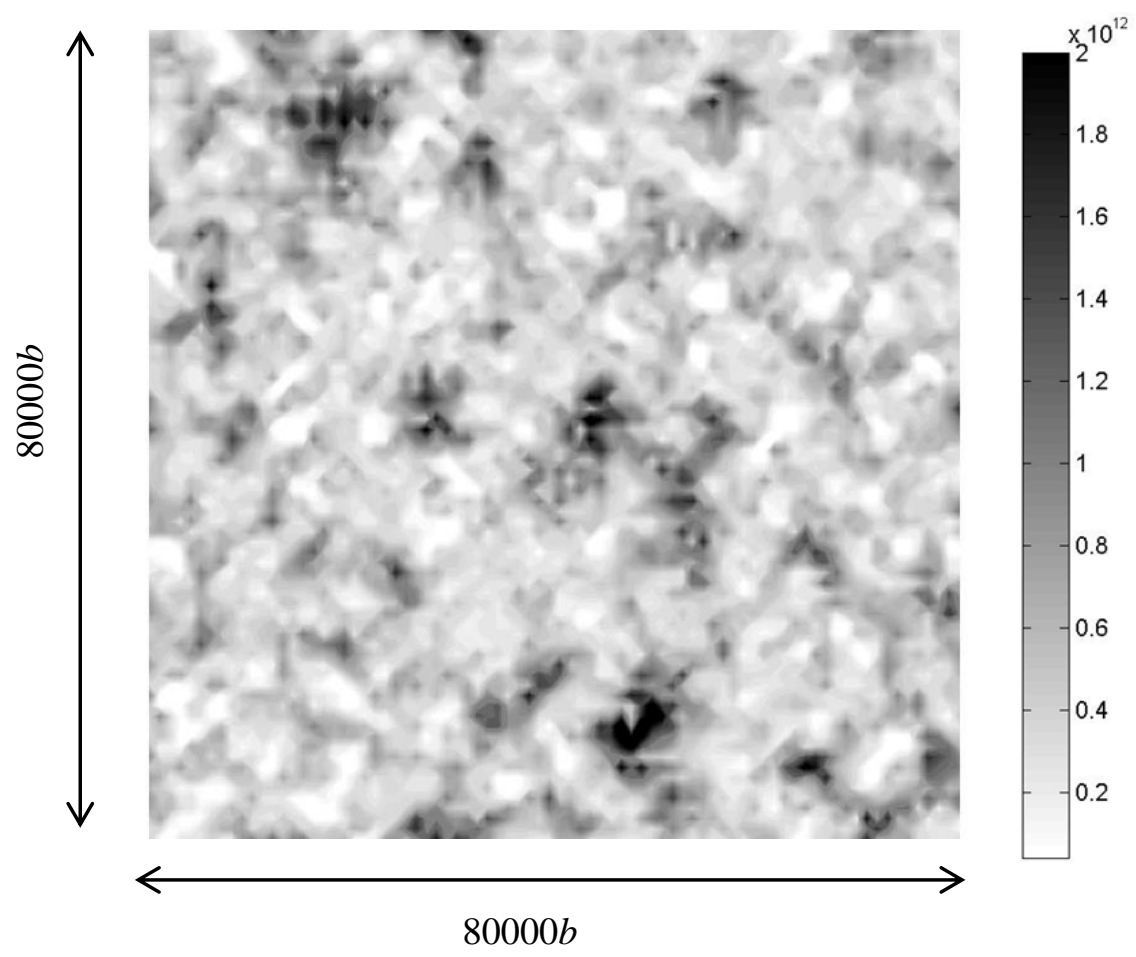

(a)

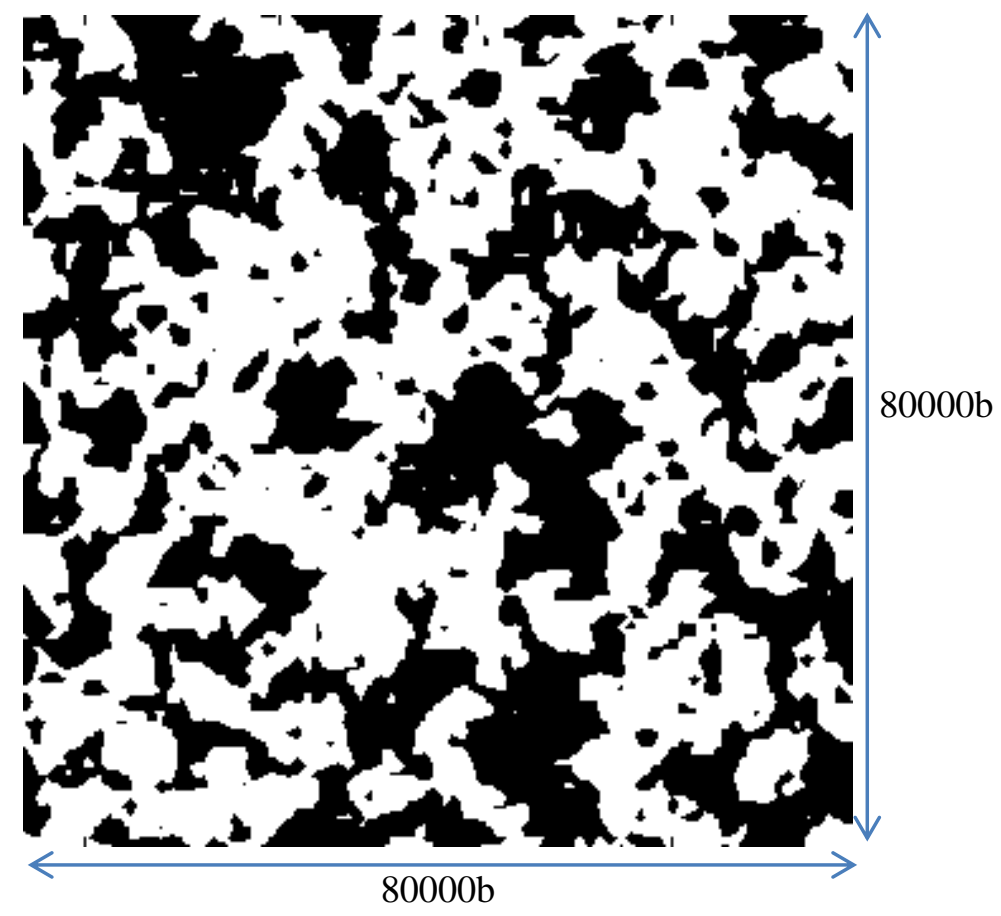

(b) 


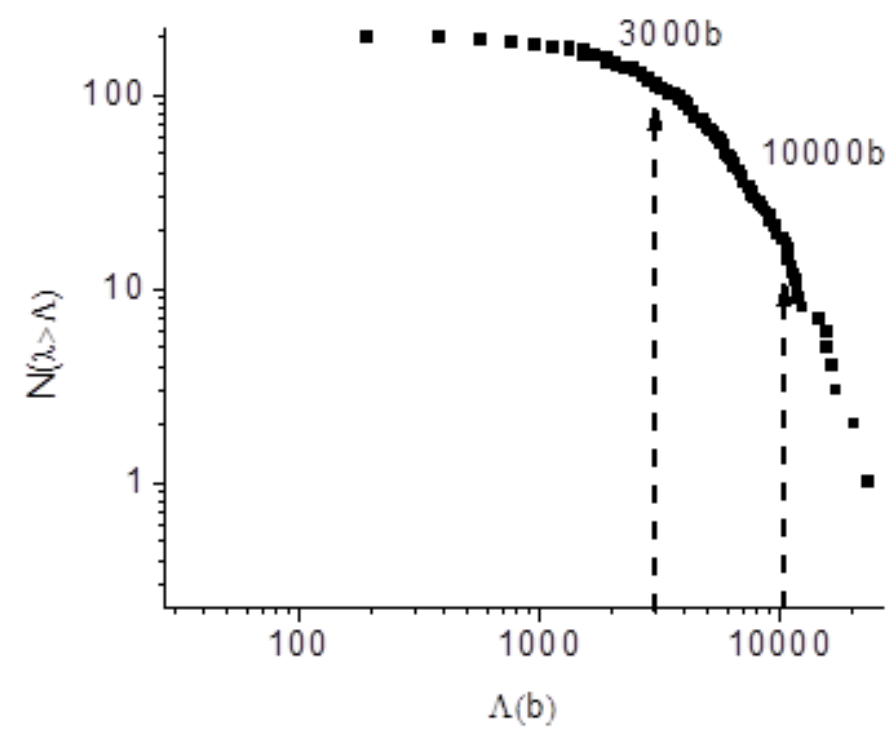

(c)

Figure 4 - Master dislocation-density microstructure from which simulation specimens of various smaller sizes are cropped to allow an ensemble study to be carried out. The spatially averaged dislocation density is $1 \times 10^{12} \mathrm{~m}^{-2}$, and the specimen dimensions are $80000 \mathrm{~b} \times$ $80000 b \times 5000 b$, corresponding to $400 \times 400 \times 25$ voxels. (a) Dislocation-density map in full density scale in units of $\mathrm{m}^{-2}$. (b) Binary image with a cutoff density at the average value of $1 \times$ $10^{12} \mathrm{~m}^{-2}$, i.e. above-average density is shown in black and below-average in white. (c) Double logarithmic plot of cumulative number of cells larger than length $\Lambda$, versus $\Lambda$.

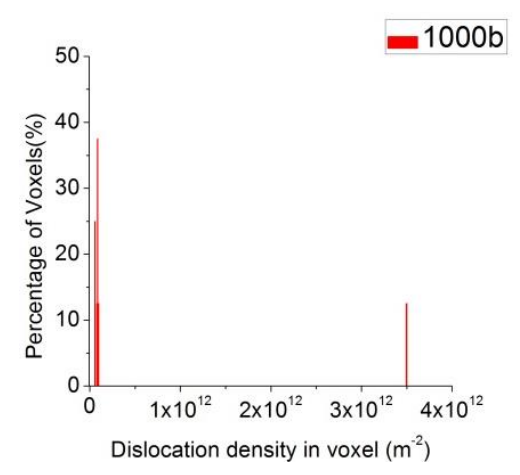

(a)

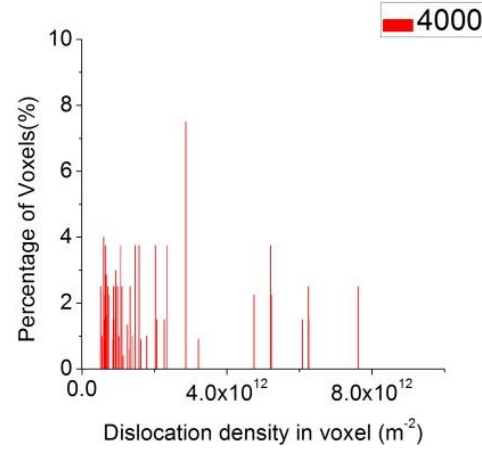

(b)

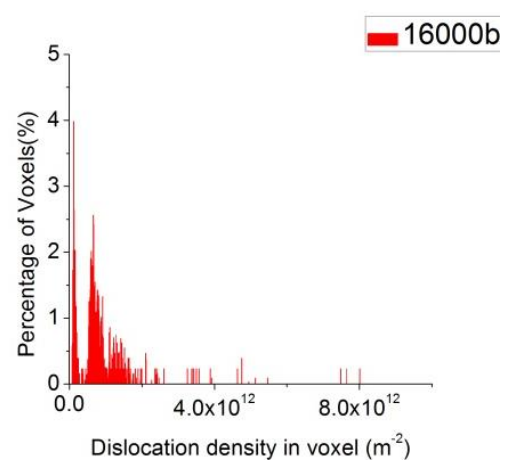

(c)

Figure 5 - Percentage of voxels of different initial densities for specimens with size (a) $1000 \mathrm{~b}$, (b) $4000 \mathrm{~b}$ and (c) $16000 \mathrm{~b}$ cropped from the master structure in Figure 4. 


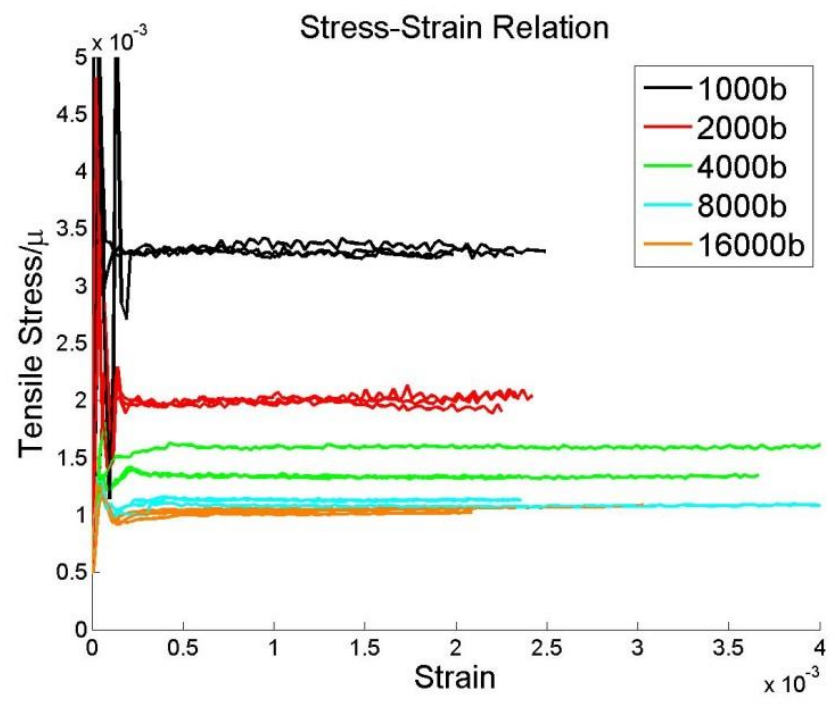

Figure 6 - Simulated stress-strain curves for various specimen sizes loaded at a constant strain rate of $2.5 \times 10^{-3} \mathrm{~s}^{-1}$. For each specimen size, three specimens were randomly cropped from the master structure in Figure 4 and their stress-strain curves were computed as shown.

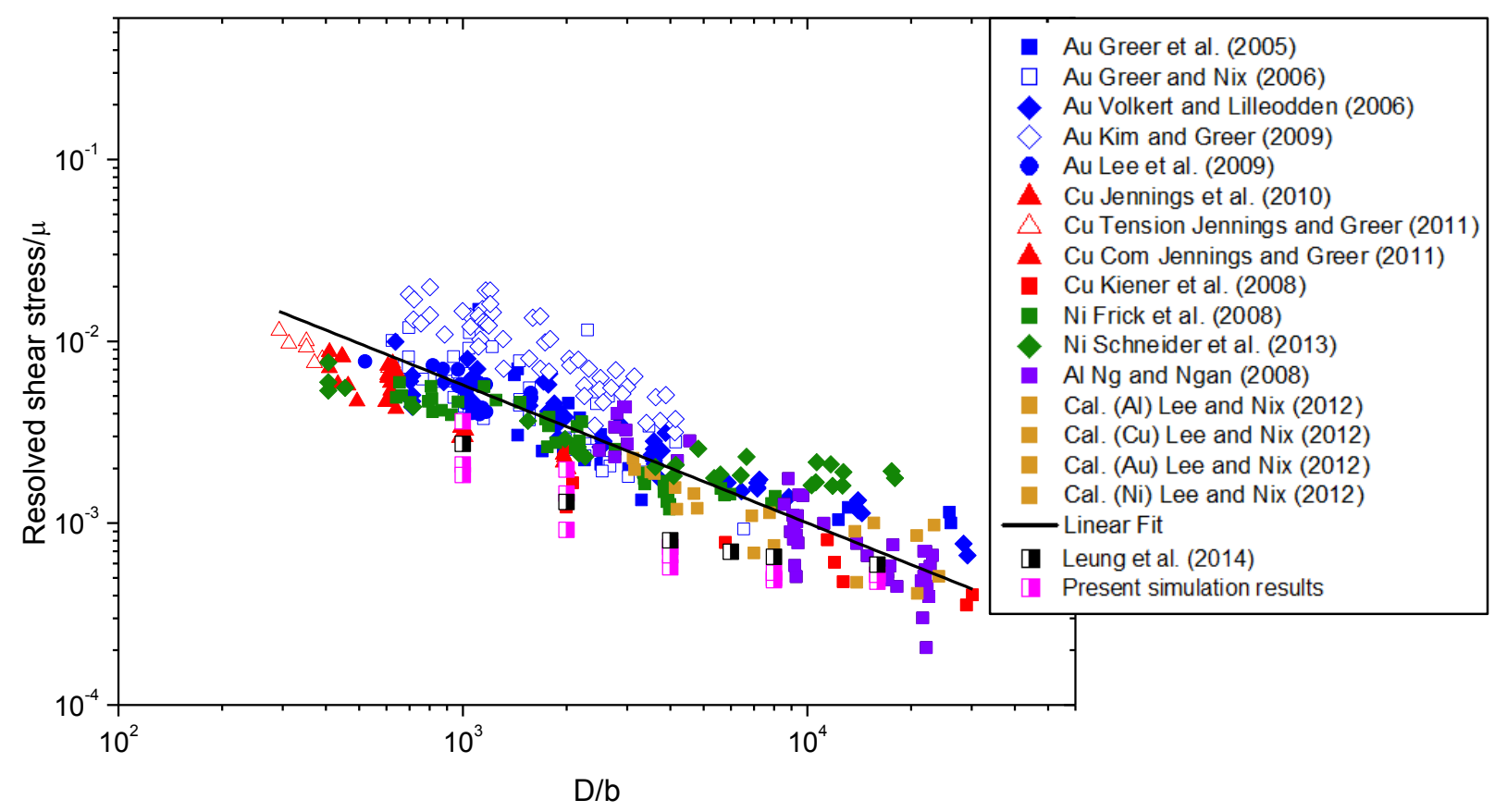

Figure 7 - Comparison of the initial yield point in the present simulations with data in the literature. The percentage or note at the end of each legend denotes the strain at which the flow 
stress was measured. The upper yield stresses in the initial yield points in Fig. 6 are used to convert into a resolved shear strength using a Schmid factor of 0.408 .

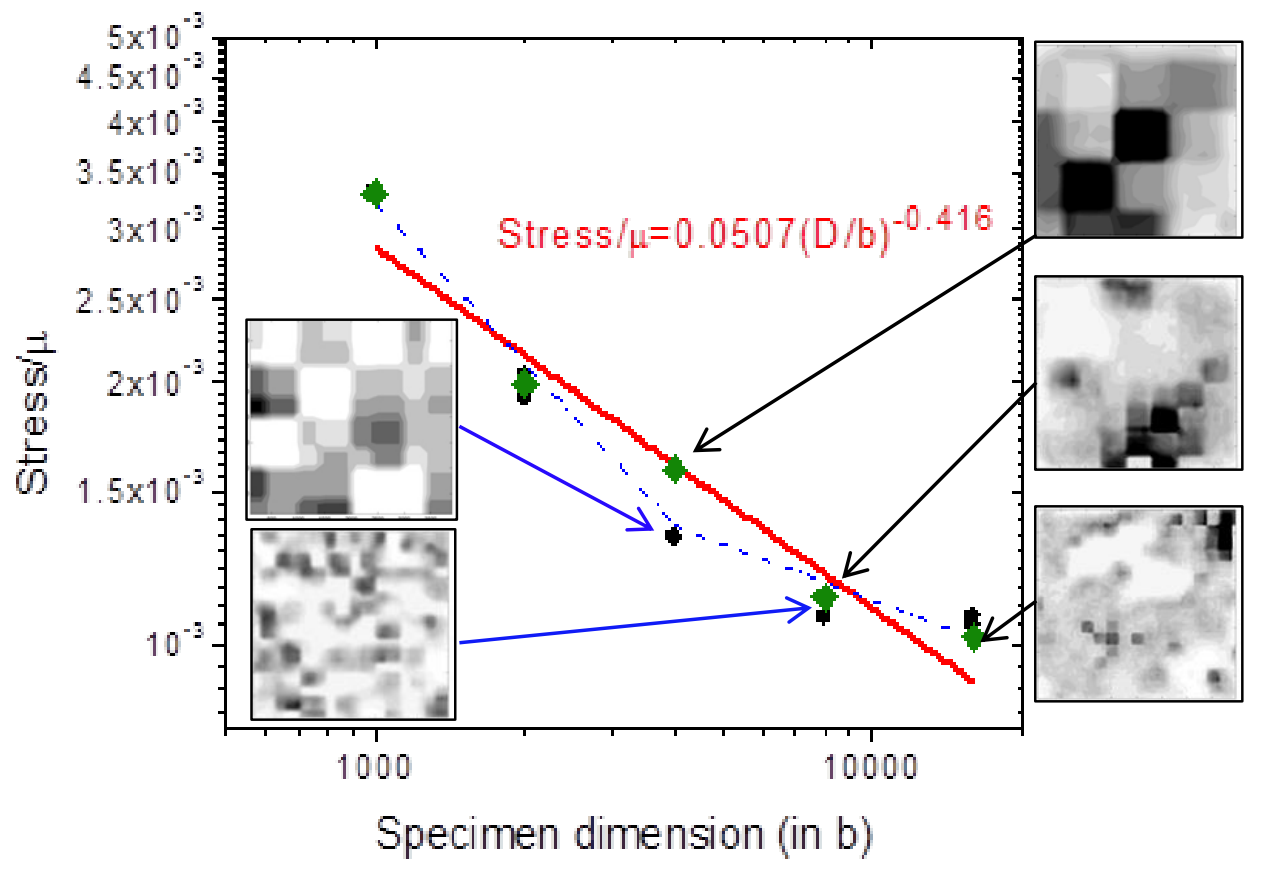

Figure 8 - Log-log plots of tensile stress at $0.2 \%$ strain normalized by shear modulus, versus normalized specimen size. Insets show the corresponding initial dislocation structures. 


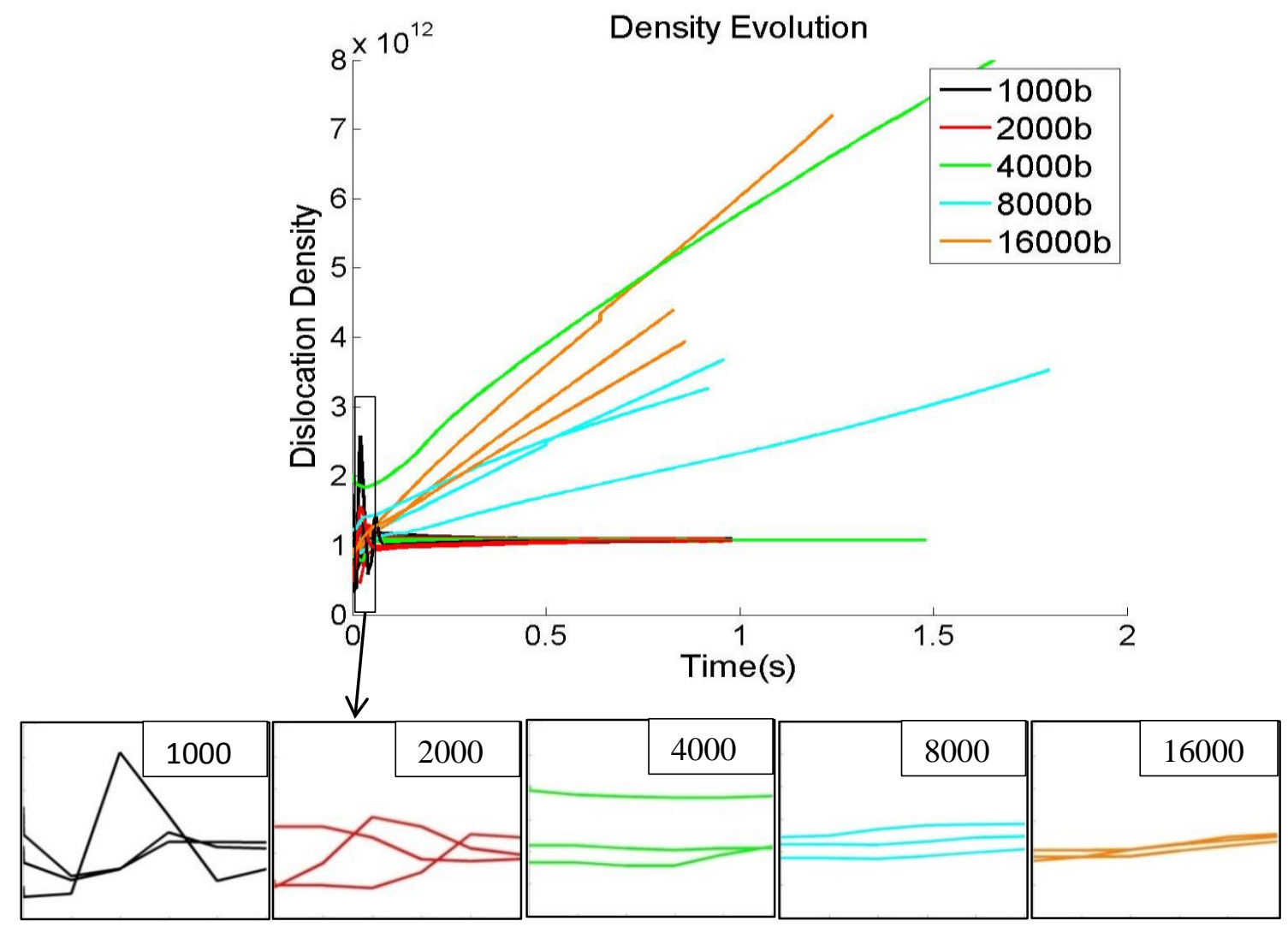

Figure 9 - Dislocation evolution of different specimen sizes. Three random samples generated from the master microstructure for each specimen size were simulated. 


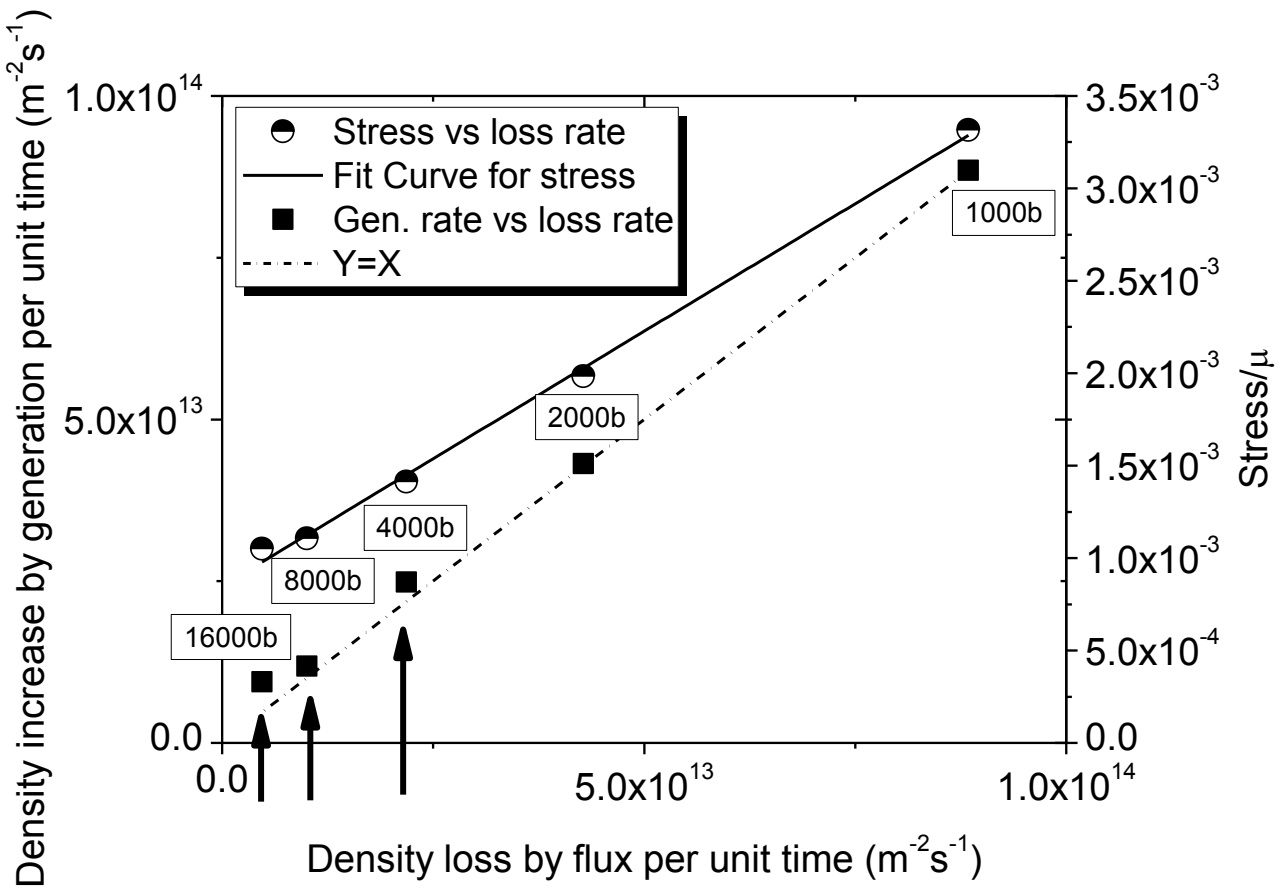

Figure 10 - Correlation between spatially averaged loss rate due to free surface, spatially averaged rate of dislocation generation and $0.2 \%$ proof stress. The data points for specimens < $4000 b$ comply with the $\mathrm{Y}=\mathrm{X}$ line, but those for specimens $>4000 b$ fall above the $\mathrm{Y}=\mathrm{X}$ line as indicated by the arrows. 


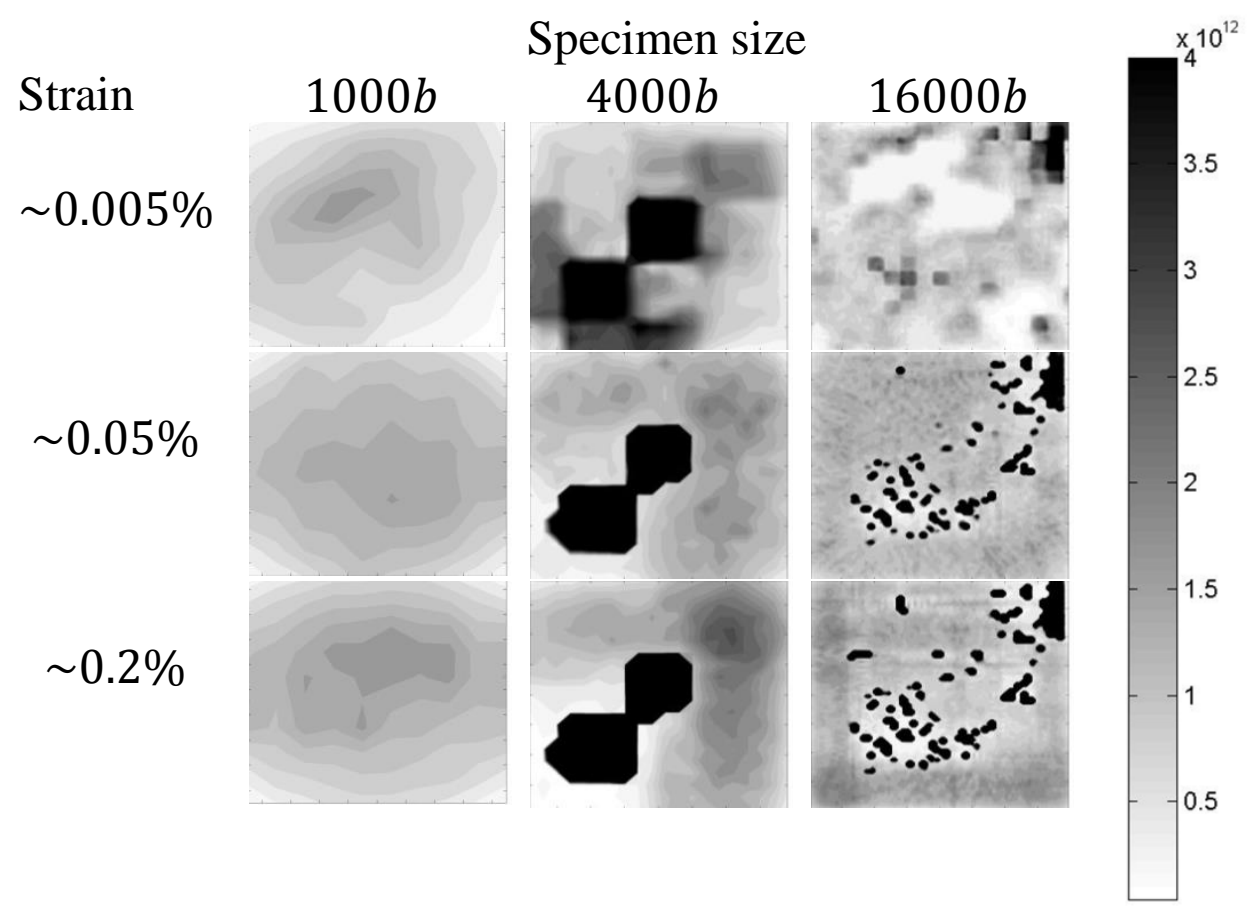

Figure 11(a) - Evolution of dislocation-density contour plots as specimens of different sizes undergo deformation. The dislocation density scale is in units of $\mathrm{m}^{-2}$.

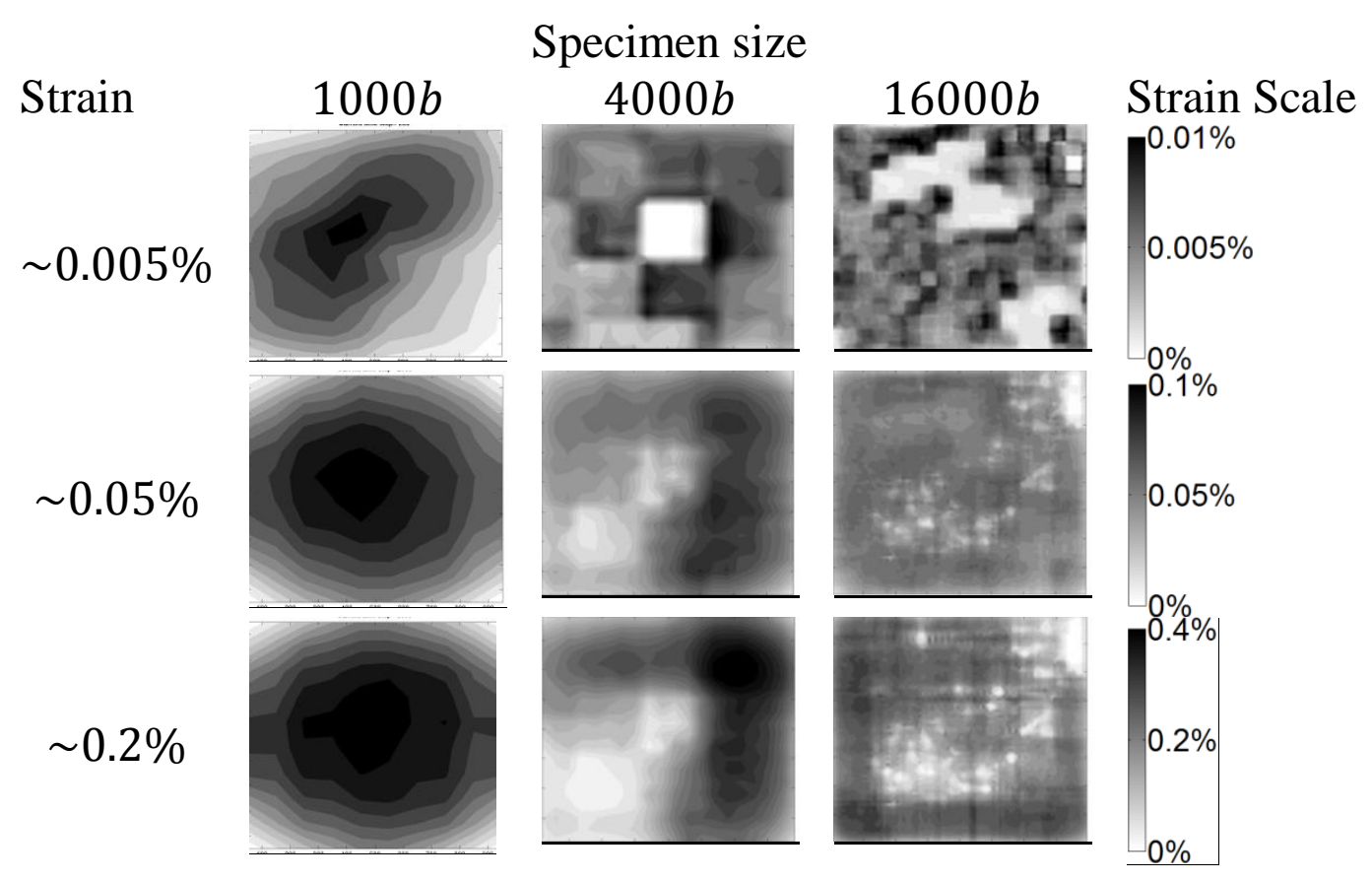

Figure 11(b) - Evolution of cumulative strain contour plots as specimens with different sizes undergo deformation. The scale of the plots is from 0 to 2 times the average specimen strain. 


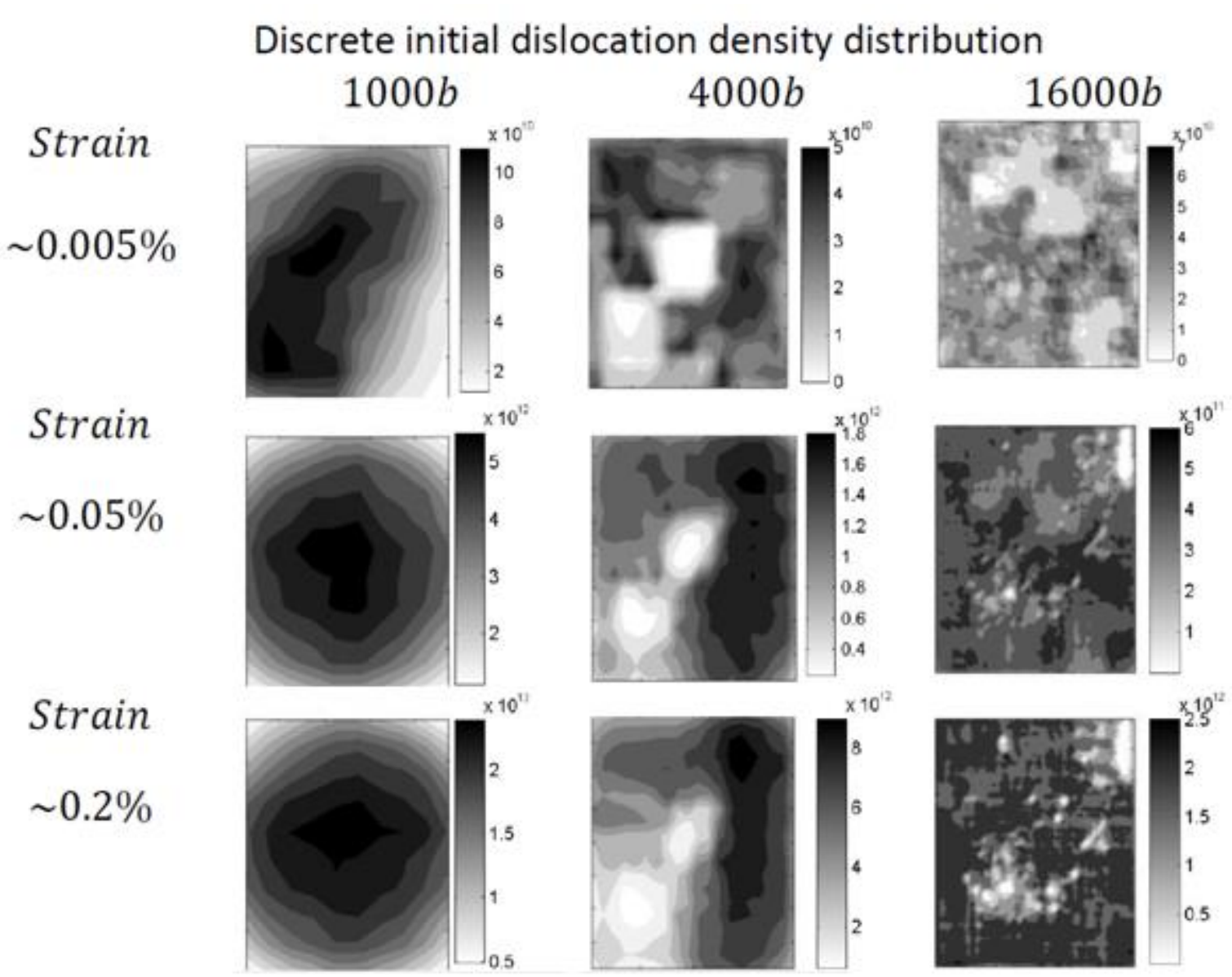

Figure 11(c) - Evolution of contour plots of the cumulative dislocation generation on the (111) slip plane as specimens with different sizes undergo deformation. The dislocation density scales are in units of $\mathrm{m}^{-2}$. 


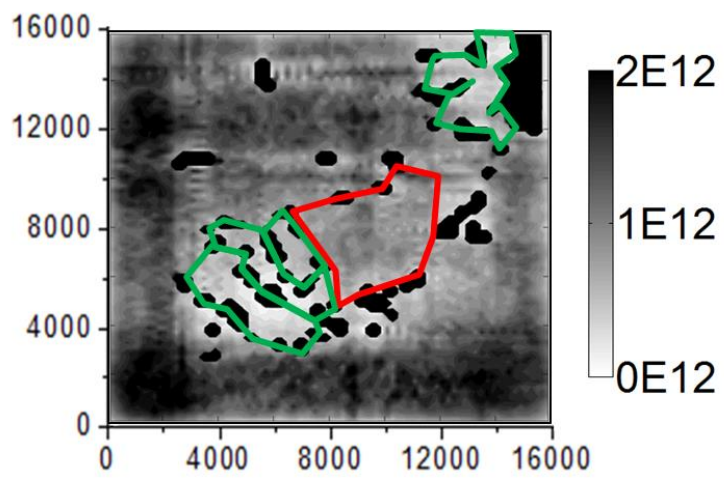

(a)

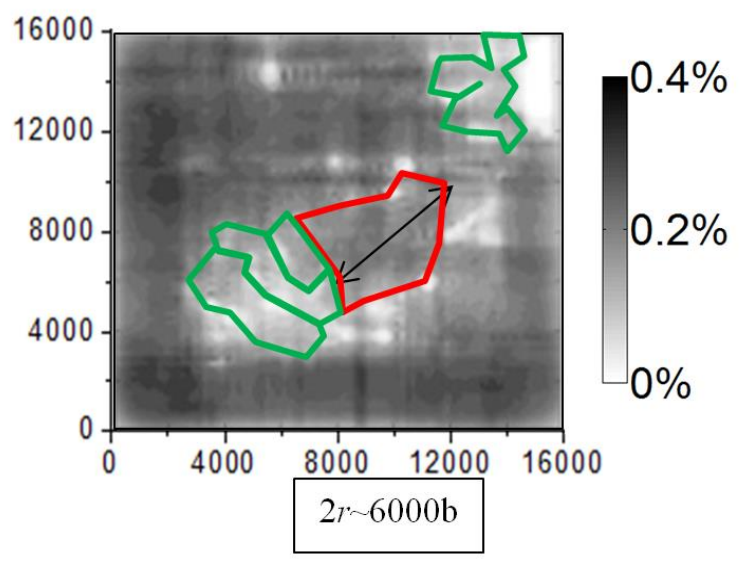

(b)

Figure 12 - Enlarged view of (a) the dislocation-density contour plot in Figure 11(a), and (b) the strain contour plot in Figure 11(b), for the $16000 \mathrm{~b}$ specimen at $\sim 0.2 \%$ strain. Red contours outline a typical soft region, with moderate dislocation density $\left(\sim 1 \times 10^{12} \mathrm{~m}^{-2}\right)$ and high strain $(\sim 0.2 \%)$, near some dense clusters. Green contours outline a few hard regions with low dislocation density and strain. Critical distance of about $2000 \mathrm{~b}$ to $3000 \mathrm{~b}$ away from dense clusters is required for the dislocation density to increase from a low level corresponding to a hard response, to a moderate level corresponding to a soft response. 


\begin{tabular}{|c|c|c|c|c|c|c|}
\hline $\begin{array}{l}\text { Specimen } \\
\text { Size }\end{array}$ & Stress $/ \mu$ & $\begin{array}{l}\text { Initial Density } \\
\left(\mathrm{m}^{-2}\right)\end{array}$ & Stress $/ \mu$ & $\begin{array}{c}\text { Initial Density } \\
\left(\mathrm{m}^{-2}\right)\end{array}$ & $\begin{array}{c}\text { Difference in } \\
\text { density }(\%)\end{array}$ & $\begin{array}{c}\text { Difference } \\
\text { in stress (\%) }\end{array}$ \\
\hline \multirow[b]{2}{*}{$1000 b$} & $3.32 \times 10^{-3}$ & $1.128 \times 10^{12}$ & $3.33 \times 10^{-3}$ & $1.739 \times 10^{12}$ & \multirow[b]{2}{*}{35.135} & \multirow[b]{2}{*}{0.185} \\
\hline & & & & & & \\
\hline \multirow[b]{3}{*}{$2000 b$} & Stress $/ \mu$ & $\begin{array}{c}\text { Initial Density } \\
\left(\mathrm{m}^{-2}\right)\end{array}$ & Stress $/ \mu$ & $\begin{array}{c}\text { Initial Density } \\
\left(\mathrm{m}^{-2}\right)\end{array}$ & $\begin{array}{c}\text { Difference in } \\
\text { density (\%) }\end{array}$ & $\begin{array}{c}\text { Difference } \\
\text { in stress (\%) }\end{array}$ \\
\hline & $1.92 \times 10^{-3}$ & $1.410 \times 10^{12}$ & $1.99 \times 10^{-3}$ & $0.595 \times 10^{12}$ & \multirow[b]{2}{*}{-137.049} & \multirow[b]{2}{*}{3.532} \\
\hline & & & & & & \\
\hline \multirow[b]{3}{*}{$4000 b$} & Stress $/ \mu$ & $\begin{array}{c}\text { Initial Density } \\
\left(\mathrm{m}^{-2}\right)\end{array}$ & Stress $/ \mu$ & $\begin{array}{c}\text { Initial Density } \\
\left(\mathrm{m}^{-2}\right)\end{array}$ & $\begin{array}{c}\text { Difference in } \\
\text { density (\%) }\end{array}$ & $\begin{array}{c}\text { Difference } \\
\text { in stress (\%) }\end{array}$ \\
\hline & $1.33 \times 10^{-3}$ & $1.100 \times 10^{12}$ & $1.59 \times 10^{-3}$ & $2.031 \times 10^{12}$ & \multirow[b]{2}{*}{45.848} & \multirow[b]{2}{*}{16.745} \\
\hline & & & & & & \\
\hline \multirow[b]{3}{*}{$8000 b$} & Stress $/ \mu$ & $\begin{array}{c}\text { Initial Density } \\
\left(\mathrm{m}^{-2}\right)\end{array}$ & Stress $/ \mu$ & $\begin{array}{l}\text { Initial Density } \\
\left(\mathrm{m}^{-2}\right)\end{array}$ & $\begin{array}{c}\text { Difference in } \\
\text { density }(\%)\end{array}$ & $\begin{array}{c}\text { Difference } \\
\text { in stress (\%) }\end{array}$ \\
\hline & $1.07 \times 10^{-3}$ & $0.906 \times 10^{12}$ & $1.13 \times 10^{-3}$ & $1.226 \times 10^{12}$ & \multirow[b]{2}{*}{26.126} & \multirow[b]{2}{*}{5.258} \\
\hline & & & & & & \\
\hline \multirow[b]{3}{*}{$16000 b$} & Stress $/ \mu$ & $\begin{array}{c}\text { Initial Density } \\
\left(\mathrm{m}^{-2}\right)\end{array}$ & Stress $/ \mu$ & $\begin{array}{c}\text { Initial Density } \\
\left(\mathrm{m}^{-2}\right)\end{array}$ & $\begin{array}{c}\text { Difference in } \\
\text { density (\%) }\end{array}$ & $\begin{array}{c}\text { Difference } \\
\text { in stress (\%) }\end{array}$ \\
\hline & $1.02 \times 10^{-3}$ & $0.879 \times 10^{12}$ & $1.08 \times 10^{-3}$ & $1.045 \times 10^{12}$ & \multirow[b]{2}{*}{15.879} & \multirow[b]{2}{*}{5.162} \\
\hline & & & & & & \\
\hline
\end{tabular}

Figure 13 - Comparison of $0.2 \%$ proof stress and initial dislocation density for specimens of the same size cropped from the master structure in Figure 4. 


\begin{tabular}{|c|c|c|c|c|c|c|}
\hline & $16000 b(1)$ & $16000 b(2)$ & $8000 b$ & $4000 b$ & $2000 b$ & $1000 b$ \\
\hline $\begin{array}{c}\text { Low initial dislocation } \\
\text { density }\end{array}$ & $\begin{array}{c}79.96 \% * \\
\left(0.88 \times 10^{12} \mathrm{~m}^{-2}\right)\end{array}$ & $\begin{array}{c}79.96 \% * \\
\left(0.88 \times 10^{12} \mathrm{~m}^{-2}\right)\end{array}$ & $\begin{array}{c}89.25 \% \\
\left(0.93 \times 10^{12} \mathrm{~m}^{-2}\right)\end{array}$ & $\begin{array}{c}86.8 \% \\
\left(1.08 \times 10^{12} \mathrm{~m}^{-2}\right)\end{array}$ & $\begin{array}{c}83.00 \% \\
\left(0.59 \times 10^{12} \mathrm{~m}^{-2}\right)\end{array}$ & $\begin{array}{c}84.00 \% \\
\left(0.50 \times 10^{12} \mathrm{~m}^{-2}\right)\end{array}$ \\
\hline $\begin{array}{c}\text { High initial dislocation } \\
\text { density }\end{array}$ & $\begin{array}{c}81.09 \% * \\
\left(0.97 \times 10^{12} \mathrm{~m}^{-2}\right)\end{array}$ & $\begin{array}{c}68.08 \% * \\
\left(1.56 \times 10^{12} \mathrm{~m}^{-2}\right)\end{array}$ & $\begin{array}{c}74.40 \% * \\
\left(1.23 \times 10^{12} \mathrm{~m}^{-2}\right)\end{array}$ & $\begin{array}{c}66.75 \% * \\
\left(2.03 \times 10^{12} \mathrm{~m}^{-2}\right)\end{array}$ & $\begin{array}{c}84.40 \% \\
\left(1.40 \times 10^{12} \mathrm{~m}^{-2}\right)\end{array}$ & $\begin{array}{c}83.70 \% \\
\left(1.35 \times 10^{12} \mathrm{~m}^{-2}\right)\end{array}$ \\
\hline $\begin{array}{c}\text { Difference } \\
(\% \text { high } \rho-\% \text { low } \rho)\end{array}$ & $1.13 \%$ & $-11.88 \%$ & $-14.85 \%$ & $-20.05 \%$ & $1.40 \%$ & $-0.30 \%$ \\
\hline
\end{tabular}

Table 1 - Percentage of soft voxels satisfying $\varepsilon_{\text {voxel }}>70 \% \varepsilon_{\text {total }}$, where $\varepsilon_{\text {total }}$ here is $0.2 \%$. Asterisk $(*)$ indicates dense dislocation clusters are present in the initial microstructure. 